\title{
Evidences for two scales in hadrons
}

\author{
B.Z. Kopeliovich, ${ }^{1,2}$ I. K. Potashnikova, ${ }^{1}$ B. Povh, ${ }^{3}$ and Ivan Schmidt ${ }^{1}$ \\ ${ }^{1}$ Departamento de Física y Centro de Estudios Subatómicos, Universidad Técnica Federico Santa María, Casilla 110-V, \\ Valparaíso, Chile \\ ${ }^{2}$ Joint Institute for Nuclear Research, Dubna, Russia \\ ${ }^{3}$ Max-Planck-Institut für Kernphysik, Postfach 103980, 69029 Heidelberg, Germany
}

(Received 27 August 2007; published 16 November 2007)

\begin{abstract}
Some unusual features observed in hadronic collisions at high energies can be understood assuming that gluons in hadrons are located within small spots occupying only about $10 \%$ of the hadrons' area. Such a conjecture about the presence of two scales in hadrons helps to explain the following: why diffractive gluon radiation is so suppressed; why the triple-Pomeron coupling shows no $t$ dependence; why total hadronic cross sections rise so slowly with energy; why diffraction cones shrink so slowly, and why $\alpha_{\mathbb{P}}^{\prime} \ll$ $\alpha_{\mathbb{R}}^{\prime}$; why the transition from hard to soft regimes in the structure functions occurs at rather large $Q^{2}$; why the observed Cronin effect at collider energies is so weak; why hard reactions sensitive to primordial parton motion (direct photon, Drell-Yan dileptons, heavy flavors, back-to-back dihadrons, seagull effect, etc.) demand such a large transverse momenta of the projectile partons, which is not explained by next-toleading order calculations; why the onset of nuclear shadowing for gluons is so delayed compared to quarks; and why shadowing is so weak.
\end{abstract}

DOI: 10.1103/PhysRevD.76.094020

PACS numbers: 12.38.Aw, 12.38.Bx, 12.38.Qk, 12.40.Nn

\section{INTRODUCTION}

The first manifestations of the gluon contribution to the total hadronic cross sections go back to the early 1970s when cross sections rising with energy were observed in intersecting storage rings (ISR) experiments. Indeed, treating a hadron as a bound state in nonrelativistic quantum mechanics, its transverse size is invariant relative to longitudinal boosts, and therefore one may expect a constant cross section at high energies (Pomeranchuk limit). Rising cross sections require new degrees of freedom, which were attributed to the emission of gluons.

The radiation of gluons is quite different from photon radiation. Gluons can radiate other gluons with the same probabilities at any rapidity, which makes it easier for gluons to cover the large rapidity interval between the colliding hadrons. On the contrary, photon radiation vanishes exponentially at large rapidity intervals from the source. Because of integration over phase space, the radiation of each gluon brings a power of $\ln (s)$ to the cross section (BFKL regime [1]). Another clear difference between QCD and QED is that the non-Abelian nature of the interactions results in elastic hadronic amplitudes which are nearly imaginary (nearly real in QED).

One may wonder, however, why the observed rise of the total hadronic cross sections is so slow, approximately $s^{0.1}$ ? The total cross section is dominated by soft interactions, so the QCD coupling is large, and a steep energy dependence is, in principle, expected. Some dynamics must therefore suppress gluon radiation. This observation might have been a first hint at the idea of the existence of a semihard scale in hadrons, which controls and suppresses gluon radiation.
Another hint to this phenomenon came in about the same period of time, from data on diffraction. It was discovered that diffractive gluon radiation is quite suppressed compared to simple expectations for this soft reaction. This is the well-known problem of the smallness of the triplePomeron coupling, which is a direct indication of the presence of a semihard scale.

Thus, data suggest an enhanced Fermi motion of gluons in light hadrons compared to the inverse hadronic radius. Correspondingly, gluons in the hadron should be located within spots of a small size. This picture was suggested in [2], and the mean radius of the spots was fitted to diffractive data at $r_{0}=0.3 \mathrm{fm}$. Therefore the area of a gluonic spot is an order of magnitude smaller than the area of the hadron.

This observation is in accord with nonperturbative QCD models. For instance, a small gluon correlation radius $0.35 \mathrm{fm}$ is a result of lattice calculations [3,4], and it is also predicted by the instanton model [5], related in this case to the instanton size $0.3 \mathrm{fm}$. Phenomenological applications of this effect were discussed in [6]. The smallness of the mean radius of gluon distribution in the proton was also confirmed by an analysis of hadronic matrix elements of the gluonic contribution to the energy momentum tensor, using QCD sum rules. It gave a value of $0.3 \mathrm{fm}$ for the radius of the corresponding gluonic form factor [7].

In this paper we present several experimental facts, which so far have not had a clear interpretation, but which can be explained assuming the location of gluons within small spots. In Sec. II we demonstrate that diffractive gluon bremsstrahlung is strongly suppressed, and that this fact is important evidence for the smallness of gluonic spots. Such a conjecture also explains an unusual feature of the 
triple-Pomeron coupling, which is the absence of a form factor.

In Sec. III we relate the smallness of the energy slope, $\epsilon=d \ln \left(\sigma_{\text {tot }}\right) / d \ln (s)=0.1$, to the gluonic semihard scale in the proton.

In Sec. IV we consider the long-standing problem of the smallness of the Pomeron trajectory slope, $\alpha_{\mathbb{P}}^{\prime}=$ $0.25 \mathrm{GeV}^{-2}$, compared to Regge trajectories, $\alpha_{\mathbb{R}}^{\prime}=$ $0.9 \mathrm{GeV}^{-2}$. Within the same model of gluonic spots we predict an even smaller value $\alpha_{\mathbb{P}}^{\prime}=0.1 \mathrm{GeV}^{-2}$, which indeed was observed in electroproduction of vector mesons. Although the effective slope of the Pomeron trajectory observed in $p p$ collisions, $\alpha_{\mathbb{D}}^{\prime}=0.25 \mathrm{GeV}^{-2}$, is larger than expected, this is explained well by the saturation of unitarity. Within the same model we reproduced well the large value of the slope of Regge trajectories, $\alpha_{\mathbb{R}}^{\prime} \approx 1 \mathrm{GeV}^{-2}$.

In Sec. V we study the manifestations of the two hadronic scales in the transition from hard to soft regimes in deeply inelastic scattering (DIS). We conclude that the observed slowing down towards the soft limit and final drop of the logarithmic $Q^{2}$ derivative of the structure function is related not to the phenomenon of saturation expected at small $x$, but to the reduction of $Q^{2}$ correlated with $x$ in data.

Naturally, gluons located within small spots have an enhanced Fermi motion. In Sec. VI we list experimental evidences for an increased primordial transverse momenta of gluons in projectile hadrons. One of the consequences is a very weak Cronin effect, predicted in [8] and successfully verified at the Relativistic Heavy Ion Collider (RHIC).

Localization of gluons within small spots also leads to dramatic changes in nuclear effects of coherence, considered in Sec. VII, such as gluon shadowing and color glass condensate, which are expected to be much weaker than usually believed. This is a result of the rather small overlap of the gluonic spots in the transverse plane. Increased transverse momentum of gluons also makes them effectively heavier. This results in a reduction by an order of magnitude of the coherence time related to gluon shadowing, and delays the onset of shadowing of gluons compared to quarks.

Some of the listed results have already been published in conference proceedings $[9,10]$.

\section{GLUON RADIATION SUPPRESSION}

If gluons in hadrons are located in small spots of radius $r_{0}$, they have enhanced transverse momenta $q_{T} \sim 1 / r_{0}$. For soft interactions it is difficult to resolve such gluons and shake them off; i.e. the gluon bremsstrahlung cross section should be suppressed compared to perturbative estimates. However, in the case of an inelastic collision followed by multiparticle production, the events with or without gluon radiation look alike. Indeed, a color-exchange interaction results in production of two flying away colored clusters of remnants of the colliding hadrons, which can create particles even if no gluons are radiated, just by means of string breaking. The produced particles build a plateau in rapidity similar to gluon bremsstrahlung, and it is therefore difficult to find any certain signature for the radiated gluons.

\section{A. Small triple-Pomeron coupling}

On the other hand, diffraction offers an exceptional possibility to identify gluon radiation. A high-energy hadron can dissociate diffractively either via excitation of the valence quark skeleton or by the radiation of gluons, and these two mechanisms are characterized by different dependence on the effective mass $\left(M_{X}\right)$ of the excitation [11],

$$
\frac{d \sigma(h p \rightarrow X p)}{d M_{X}^{2}}= \begin{cases}\frac{1}{M_{X}^{3}} & \text { quark skeleton, } \\ \frac{1}{M_{X}^{2}} & \text { gluon bremsstrahlung. }\end{cases}
$$

The $M_{X}$ dependence at large $M_{X}$ correlates with the spin of the slowest particle produced in the excitation. Only a vector particle, i.e. a gluon, can provide a $1 / M_{X}^{2}$ dependence. Thus, one can single out the large mass tail from the $M_{X}$ distribution which gives the cross section of diffractive gluon radiation.

There is a simple hint showing that the diffractive excitation cross section is unexpectedly small. In fact, it has been known since the 1970s that the triple-Pomeron coupling is quite small. To appreciate this statement one can express diffraction in terms of the Pomeron-proton total cross section, as is demonstrated pictorially in Fig. 1. Treating the Pomeron as a gluonic dipole, one may expect a cross section which is a Casimir factor 9/4 larger than that for quarkonium. Comparing with the pion-proton cross section, one arrives at about $50 \mathrm{mb}$. However, experimental results for $\sigma_{\text {tot }}^{\mathbb{P} p}\left(s^{\prime}=M_{X}^{2}\right)$, depicted in Fig. 2, give quite a smaller value, less than $2 \mathrm{mb}$ at large $M_{X}^{2}$, where the triplePomeron term is expected to dominate. At smaller invariant masses $M_{X}$ the triple-Regge term $\mathbb{P P R}$ is responsible for the contribution that decreases with decreasing $M_{X}$.

The Pomeron-Pomeron total cross section measured in double diffractive processes is plotted in Fig. 3. It turns out that $\sigma_{\mathrm{tot}}^{\mathbb{P P}}$ is even an order of magnitude smaller than $\sigma_{\mathrm{tot}}^{\mathbb{P} p}$.

Of course, a glue-glue dipole should interact more strongly than a $\bar{q} q$ dipole of the same size. However, if the former has a smaller transverse size, then its interaction

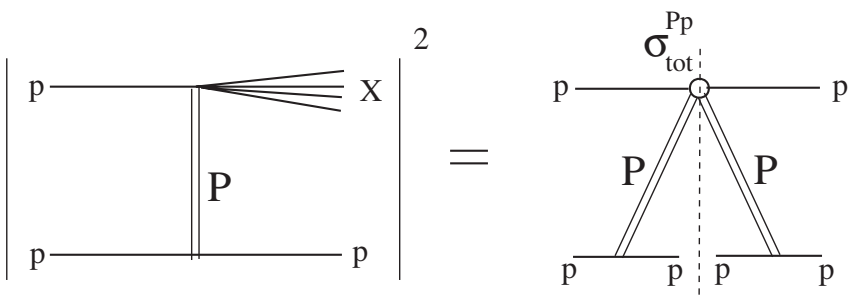

FIG. 1. The cross section of diffractive excitation of a proton expressed in terms of the total Pomeron-proton cross section. 


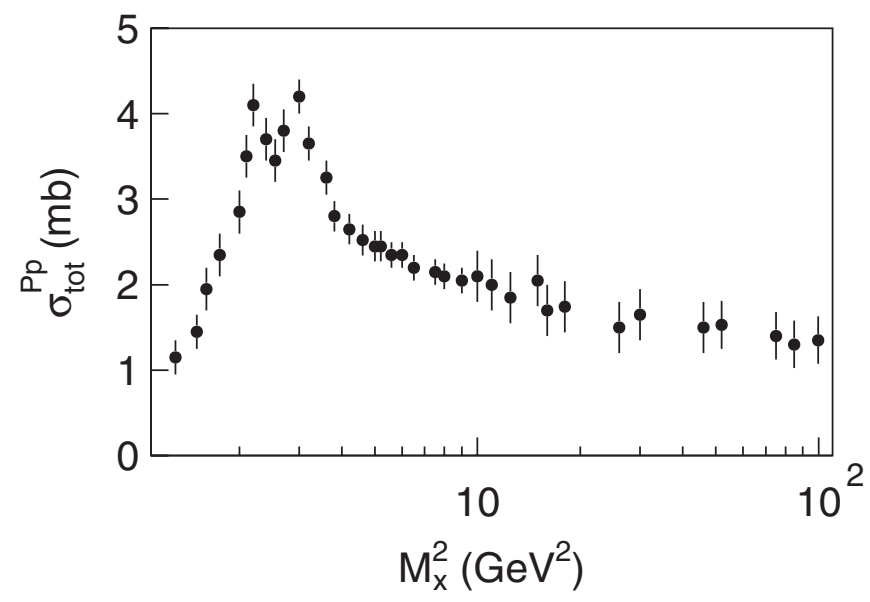

FIG. 2. Pomeron-proton total cross sections $\sigma_{\text {tot }}^{\mathbb{P} p}$ as a function of invariant mass squared, $s^{\prime}=M_{X}^{2}$, extracted from data on $p p \rightarrow p X[56]$.

is subject to color transparency [12] and the cross section may be very small. Therefore a straightforward explanation for the above dramatic disagreement would be a much smaller size of the gluonic dipole (Pomeron) compared to the quark-antiquark dipole (pion), and as a consequence one concludes that gluons should be located within small spots in the proton. Moreover, making the simple conjecture that the cross section is $\pi R_{\mathbb{D}}^{2}$, the Pomeron radius was found in [13] to be $R_{\mathbb{P}}^{2}=0.01 \mathrm{fm}^{2}$, which is an order of

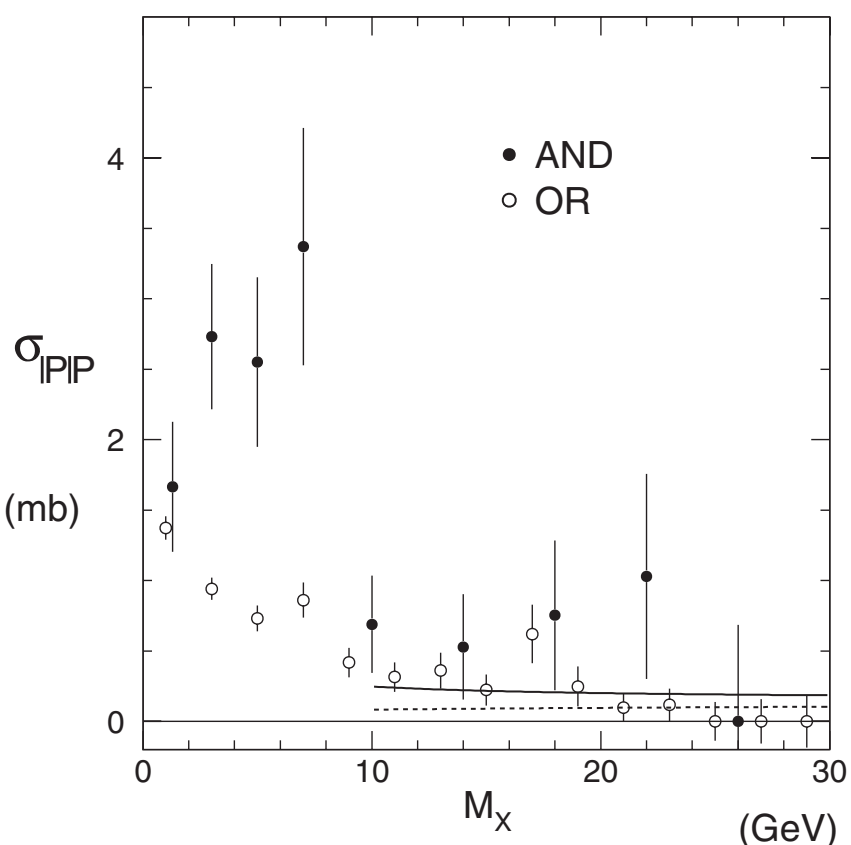

FIG. 3. The total Pomeron-Pomeron cross section measured in double diffractive processes [57]. Samples labeled "AND" and "OR" correspond to different criteria of event selection. The solid and dashed curves correspond to expectations based on Regge factorization [57]. magnitude smaller than the result of [2] and the evaluations done in this paper.

The above argumentation is rather speculative, since the possibility to describe diffraction in terms of a Pomeron flux is questionable beyond the Regge-pole model. A more consistent approach would be a calculation of gluon radiation caused by a colorless gluonic exchange (Pomeron). If Weizsäcker-Williams gluons in the proton are located within small spots which are hardly resolved by soft interactions, bremsstrahlung will be suppressed. The mean transverse size of these spots was fitted in [2] to single diffraction data with large effective masses and found to be $\left\langle r_{T}\right\rangle \sim r_{0}=0.3 \mathrm{fm}$. Such gluons have much more intensive Fermi motion than massless perturbative ones, and they are less sensitive to an external kick; i.e. gluon radiation is suppressed.

Let us start by calculating the cross section of diffractive gluon radiation with a high-energy quark interacting with a nucleon. For this purpose we need the light-cone wave function of the quark-gluon Fock component of a quark, which was calculated in [2] within a model describing the nonperturbative interaction of gluons via a phenomenological light-cone potential taken in an oscillatory form. The result reads

$$
\Psi_{q g}\left(\vec{r}_{T}\right)=-\frac{2 i}{\pi} \sqrt{\frac{\alpha}{3}} \frac{\vec{r}_{T} \cdot \vec{e}^{*}}{r_{T}^{2}} \exp \left(-\frac{r_{T}^{2}}{2 r_{0}^{2}}\right)
$$

Here $r_{T}$ is the transverse quark-gluon separation; $\vec{e}$ is the gluon polarization vector. We assume that the gluon, which is a vector particle, is carrying a negligible fraction $\alpha \ll 1$ of the quark momentum.

Of course, the concrete shape of the light-cone potential is not crucial; what is important is the smallness of the mean quark-gluon separation. Alternatively, one can rely on the perturbative light-cone distribution amplitude for the quark-gluon fluctuation, but assuming the gluon to be effectively heavy. The corresponding light-cone distribution amplitude for radiation of a transversely polarized gluon with $\alpha \ll 1$ reads [2,14]

$$
\Psi_{q g}\left(\vec{r}_{T}\right)=-\frac{2 i}{\pi} \sqrt{\frac{\alpha_{s}}{3}} \frac{\vec{r}_{T} \cdot \vec{e}^{*}}{r_{T}} m_{g} K_{1}\left(r_{T} m_{g}\right) .
$$

With the effective gluon mass $m_{g} \approx 0.7 \mathrm{GeV}$ the mean quark-gluon transverse separations with Eqs. (2) and (3) are similar. Thus, either Eq. (2) or Eq. (3) can be viewed as a source of small gluonic spots in the proton.

Once the light-cone quark-gluon distribution function is known, one can calculate the cross section of diffractive gluon radiation by a high-energy quark interaction with a nucleon [2], 


$$
\begin{aligned}
& \left.\frac{d \sigma(q N \rightarrow q G N)}{d x_{F} d p_{T}^{2}}\right|_{p_{T}=0} \\
& =\frac{1}{16 \pi\left(1-x_{F}\right)} \int d^{2} r_{T}\left|\Psi_{q G}\left(\vec{r}_{T}\right)\right|^{2} \tilde{\sigma}^{2}\left(r_{T}, s\right) .
\end{aligned}
$$

Here the cross section $\tilde{\sigma}\left(r_{T}, s\right)$ is not just the cross section of the interaction of a $q G$ dipole. In fact, this dipole is not even colorless. As usual, diffractive excitation is possible due to the difference between elastic amplitudes for different Fock states, in this case the bare quark $|q\rangle_{0}$ and the $|q G\rangle$ pair. Since they have the same color, the difference emerges from the color-dipole moment of the $q-G$ pair. It is shown in [2] (see, in particular, Appendix A.2) that $\tilde{\sigma}\left(r_{T}, s\right)=\frac{9}{8} \sigma_{\bar{q} q}\left(r_{T}, s\right)$, where $\sigma_{\bar{q} q}\left(r_{T}, s\right)$ is the cross section for the interaction of a nucleon with a $q \bar{q}$ dipole of transverse separation $r_{T}$.

We use the saturated form of this energy dependent dipole cross section, suitable for the soft processes under consideration,

$$
\sigma_{\bar{q} q}\left(r_{T}, s\right)=\sigma_{0}(s)\left[1-\exp \left(-\frac{r_{T}^{2}}{R_{0}^{2}(s)}\right)\right]
$$

where $R_{0}(s)=0.88 \mathrm{fm}\left(s_{0} / s\right)^{0.14}$ and $s_{0}=1000 \mathrm{GeV}^{2}$ [2]. The energy dependent factor $\sigma_{0}(s)$ is defined as

$$
\sigma_{0}(s)=\sigma_{\mathrm{tot}}^{\pi p}(s)\left(1+\frac{3 R_{0}^{2}(s)}{8\left\langle r_{\mathrm{ch}}^{2}\right\rangle_{\pi}}\right)
$$

where $\left\langle r_{\mathrm{ch}}^{2}\right\rangle_{\pi}=0.44 \pm 0.01 \mathrm{fm}^{2}$ is the mean square of the pion charge radius. The $s$-dependent dipole cross section, Eq. (5), is fitted [2] to data for hadronic cross sections, for real photoproduction and also for low- $Q^{2}$ HERA data for the proton structure function. The cross section (5) averaged with the pion wave function squared (see below) automatically reproduces the pion-proton cross section.

The next step is to integrate over $p_{T}$ the cross section of diffractive gluon radiation provided that the forward one, Eq. (4), is known. In terms of Regge phenomenology, diffractive radiation corresponds to the triple-Pomeron term in the cross section of single diffraction. The data agree with a Gaussian $p_{T}$ dependence of the triplePomeron term with the slope [11]

$$
B_{3 \mathbb{P}}^{p p}\left(x_{F}\right)=B_{3 \mathbb{P}}^{0}+2 \alpha_{\mathbb{P}}^{\prime} \ln \left(\frac{1}{1-x_{F}}\right),
$$

where $B_{3 \mathbb{P}}^{0}=4.2 \mathrm{GeV}^{-2}$ and $\alpha_{\mathbb{P}}^{\prime}=0.25 \mathrm{GeV}^{-2}$.

Now we are in a position to evaluate the effective triplePomeron part of the single diffraction cross section for $p p$ collisions, employing the wave function, Eq. (2), and the saturated shape for the cross section, Eq. (5),

$$
\begin{aligned}
{\left[\frac{d \sigma(p p \rightarrow p X)}{d x_{F} d p_{T}^{2}}\right]_{3 \mathbb{P}}=} & \frac{81 \alpha_{s} \sigma_{0}^{2}(s)}{(16 \pi)^{2}\left(1-x_{F}\right)} \ln \left[1+\frac{\epsilon^{2}(s)}{1+2 \epsilon(s)}\right] \\
& \times \exp \left[-p_{T}^{2} B_{3 \mathbb{P}}^{p p}\left(x_{F}\right)\right],
\end{aligned}
$$

where $\epsilon(s)=r_{0}^{2} / R_{0}^{2}(s)$. At high energies $\epsilon(s)$ is rather small, and then the single diffractive cross section, Eq. (8), is proportional to $r_{0}^{4}$. This is why this process is quite sensitive to the value of $r_{0}$, and therefore provides an efficient way to determine the size of gluonic spots in the proton, $r_{0} \approx 0.3 \mathrm{fm}$ [2].

Notice that the interference between the diffractive amplitudes of gluon radiation by different quarks in the proton should not be appreciable, since $r_{0}$ is small compared to the proton radius. Explicit calculations performed in [2] confirm this.

\section{B. Unitarity corrections}

Any large rapidity gap (LRG) process is subject to unitarity or absorptive corrections, which may be substantial. Indeed, initial/final state interactions tend to fill the rapidity gap by producing particles, and one may treat such corrections as a survival probability of the rapidity gap. Such corrections are, in general, especially large and may completely terminate the gap in the vicinity of the unitarity limit, which is also called the black disk regime. Notice that elastic scattering is an exclusion: unitarity corrections enhance it, and the elastic cross section is maximal in the black disk limit.

Since the phenomenological dipole cross section $\sigma_{\bar{q} q}(s)$ is fitted to data, we assume that it includes by default all the unitarity corrections. Thus, the amplitude of the diffractive excitation of a quark, $q N \rightarrow q G N$, does include all the absorptive corrections contained in the phenomenological dipole cross section. However, the presence of other projectile valence quarks, the spectators, should not be ignored. Indeed, any inelastic interactions of the largesize, three-quark dipole in the proton will cause particle production which will fill the rapidity gap. Thus one may expect large absorptive corrections to the cross section of diffractive gluon radiation.

The data for elastic $p p$ scattering show that the partial amplitude $f_{\mathrm{el}}^{p p}(b, s)$ hardly varies with energy at small impact parameters $b \rightarrow 0$, while it rises as a function of energy at large $b$ [15-17]. This is usually interpreted as a manifestation of saturation of the unitarity $\operatorname{limit}, \operatorname{Im} f_{\mathrm{el}}^{p p} \leq$ 1. Indeed, this condition imposes a tight restriction at small $b$, where $\operatorname{Im} f_{\mathrm{el}}^{p p} \approx 1$, leaving almost no room for further rise. Correspondingly, the amplitude of any off-diagonal process including single triple-Pomeron diffraction acquires a suppression factor

$$
f_{s d}^{p p}(b, s) \Rightarrow f_{s d}^{p p}(b, s)\left[1-\operatorname{Im} f_{\mathrm{el}}^{p p}(b, s)\right],
$$

due to unitarity or absorptive corrections. This factor, which expresses the survival probability of LRG, is known to decrease with energy [18]. The interplay of the rising and falling energy dependence of the two factors in (9) may explain the observed flat behavior of the single diffractive cross section $[19,20]$. 
Since $\operatorname{Im} f_{\mathrm{el}}^{p p}(b, s)$ is known directly from data, it would be straightforward to just fit the data with any proper parametrization and use the result in Eq. (9). Alternatively, one can use any model which provides a good description for $\operatorname{Im} f_{\mathrm{el}}^{p p}(b, s)$. It is demonstrated in [17] that even the simple model that treats the Pomeron as a Regge pole with no unitarity corrections describes reasonably well not only the total hadronic cross sections, but even the data for $f_{\mathrm{el}}^{p p}(b, s)$. In this model,

$$
\operatorname{Im} f_{\mathrm{el}}^{p p}(b, s)=\frac{\sigma_{\mathrm{tot}}^{p p}(s)}{4 \pi B_{\mathrm{el}}^{p p}(s)} \exp \left[-\frac{b^{2}}{2 B_{\mathrm{el}}^{p p}(s)}\right] .
$$

Here and for further applications we use the parametrization from [21], $\quad \sigma_{\text {tot }}^{p p}(s)=18.76 \mathrm{mb} \times\left(s / M_{0}^{2}\right)^{0.093}+$ $\sigma_{R}^{p p}(s)$, where $M_{0}=1 \mathrm{GeV}$. The Reggeon part of the cross section $\sigma_{R}^{p p}(s)$ is small at high energies and can be found in
[21]. The elastic slope is

$$
B_{\mathrm{el}}^{p p}(s)=B_{\mathrm{el}}^{0}+2 \alpha_{\mathbb{P}}^{\prime} \ln \left(s / M_{0}^{2}\right),
$$

with $B_{\mathrm{el}}^{0}=8.9 \mathrm{GeV}^{-2}$ and $\alpha_{\mathbb{P}}^{\prime}=0.25 \mathrm{GeV}^{-2}$. Note that the elastic amplitude at small impact parameters, i.e. the preexponential factor in (10), hardly changes with energy, imitating the saturation of unitarity. This fact was known back in the 1970s as a geometrical scaling. It is demonstrated in [17] (see Fig. 9) that not only at $b=0$, but in the whole range of impact parameters the model, Eq. (10), describes reasonably well the energy dependence of the partial amplitude $f_{\mathrm{el}}^{p p}(b, s)$.

Using the Fourier transformed Eq. (10) we arrive at the following cross section for single diffraction integrated over momentum transfer,

$$
\begin{aligned}
{\left[\frac{d \sigma(p p \rightarrow p X)}{d x_{F}}\right]_{3 \mathbb{P}}=} & \frac{81 \alpha_{s} \sigma_{0}^{2}(s)}{(16 \pi)^{2}\left(1-x_{F}\right) B_{3 \mathbb{P}}^{p p}\left(x_{F}\right)} \ln \left[1+\frac{\epsilon^{2}(s)}{1+2 \epsilon(s)}\right] \\
& \times\left\{1-\frac{1}{\pi} \frac{\sigma_{\mathrm{tot}}^{p p}(s)}{B_{3 \mathbb{P}}^{p p}\left(x_{F}\right)+2 B_{\mathrm{el}}^{p p}(s)}+\frac{1}{(4 \pi)^{2}} \frac{\left[\sigma_{\mathrm{tot}}^{p p}(s)\right]^{2}}{B_{\mathrm{el}}^{p p}(s)\left[B_{3 \mathbb{P}}^{p p}\left(x_{F}\right)+B_{\mathrm{el}}^{p p}(s)\right]}\right]
\end{aligned}
$$

Comparing this result with experimental data one can find the mean gluonic radius $r_{0}$. We use the triple-Regge fit [11] which gives, for the triple-Pomeron part of the cross section,

$$
\left[\frac{d \sigma(p p \rightarrow p X)}{d x_{F}}\right]_{3 \mathbb{P}}=\frac{G_{3 \mathbb{P}}(0)}{\left(1-x_{F}\right) B_{3 \mathbb{P}}^{p p}(x F)},
$$

where $G_{3 \mathbb{P}}(0)=3.2 \mathrm{mb} / \mathrm{GeV}^{2}$ is the effective triplePomeron coupling at $t=0$, including also three Pomeron-proton vertices.

We fix the QCD coupling at the scale corresponding to the mean transverse momentum of gluons, $\alpha_{s}\left(1 / r_{0}^{2}\right) \approx 0.4$ [17]. Then, comparing Eqs. (12) and (13) we arrive at $r_{0} \approx 0.3 \mathrm{fm}$.

\section{Why the triple-Pomeron vertex has no structure}

The elastic slope, Eq. (11), which is half of the $p p$ interaction radius squared, contains two terms. The second term, which depends on energy, has its origin in the Gribov's diffusion of partons in the transverse plane. The first constant term, $B_{\mathrm{el}}^{0}$, comes from the Pomeron-proton form factors of the colliding protons.

Analogously, the first term in the triple-Pomeron slope, Eq. (7), should consist of contributions coming from the form factors of both the proton and the triple-Pomeron vertex. Surprisingly, it turns out that $B_{3 \mathbb{P}}^{0} \approx \frac{1}{2} B_{\mathrm{el}}^{0}$, which is just the contribution of the target proton. Nothing is left for the triple-Pomeron vertex. This has been another known puzzle since the Regge era [11].
To evaluate how much a dipole of transverse separation $r_{0}$ contributes to the slope compared to the proton contribution, we use the mean transverse diameter of the proton squared, which equals $\frac{8}{3}\left\langle r_{\mathrm{ch}}^{2}\right\rangle$, where the mean proton charge radius squared $\left\langle r_{\mathrm{ch}}^{2}\right\rangle=0.8 \mathrm{fm}^{2}$. Then the relative correction to the slope related to the dipole size is

$$
\delta=\frac{3 r_{0}^{2}}{8\left\langle r_{\mathrm{ch}}^{2}\right\rangle}=4 \% .
$$

This very small result explains why the triple-Pomeron vertex looks structureless in data. This is another manifestation of the presence of small gluonic spots in the proton.

\section{TOTAL HADRONIC CROSS SECTIONS: WHY THE ENERGY DEPENDENCE IS SO WEAK}

It has been known since 1973 that hadronic cross sections rise with energy approximately as $s^{\epsilon}$, where the exponent is quite small, $\epsilon \approx 0.1$. What is the origin of this small number? We do not expect any small parameters in the soft regime of strong interactions.

The rise of the cross sections is related to gluon bremsstrahlung. Indeed, without gluon radiation the geometrical cross section of two hadrons would be constant, since their transverse size is Lorentz invariant, i.e. energy independent. In fact, the radiation of each gluon has a phase space proportional to $\ln (s)$, and multigluon radiation leads to powers of $\ln (s)$ in the cross section. On the other hand, we have already concluded that radiation of gluons is suppressed if they are located within small spots, since in this case it is difficult to resolve them and shake them off. 
Thus, the suppression of diffractive gluon radiation and the observed weak energy dependence of total hadronic cross sections have the same origin.

The calculations performed in [17] confirm this. The hadronic cross section was found to have the following structure,

$$
\sigma_{\mathrm{tot}}=\sigma_{0}+\sigma_{1}\left(\frac{s}{s_{0}}\right)^{\Delta}
$$

where $\sigma_{0}$ is the energy independent term, related to hadronic collisions without gluon radiation. The second term in (15) is related to the contribution of gluon bremsstrahlung to the total cross section. Since this part is expected to be small, $\sigma_{1}$ should be small. Indeed, it was found in [17] that $\sigma_{1}=27 / 4 C r_{0}^{2}$, where the factor $C \approx 2.4$ is related to the behavior of the dipole-proton cross section calculated in the Born approximation at small separations, $\sigma\left(r_{T}\right)=$ $C r_{T}^{2}$ at $r_{T} \rightarrow 0$.

The energy dependence of the second term in (15) was found to be rather steep, with an exponent $\Delta=4 \alpha_{s} / 3 \pi=$ 0.17 , which seems to be too large compared to the experimentally measured $\epsilon \approx 0.1$. There is, however, no contradiction due to the presence of the energy independent, first term in (15). Approximating the cross section (15) by the simple power dependence on energy, the effective exponent reads

$$
\epsilon=\frac{\Delta}{1+\sigma_{0} / \sigma_{1}\left(s / s_{0}\right)^{-\Delta}}
$$

So, one should expect a growing steepness of the energy dependence for the total cross section. The value of $r_{0}$ can be estimated by demanding that the effective exponent be $\epsilon \approx 0.1$ in the energy range of fixed target experiments, say, at $s \sim 1000 \mathrm{GeV}^{2}$. With $\sigma_{0}=40 \mathrm{mb}$ found in [17], one gets $r_{0}=0.3 \mathrm{fm}$.

Thus, the observed slow rise of the total hadronic cross sections provides another evidence of the existence of small gluonic spots with transverse size $r_{0} \sim 0.3 \mathrm{fm}$.

Notice that Eq. (16) may lead to a nonuniversal energy dependence, which correlates with the magnitude of the total cross section. Unfortunately, the difference between $\epsilon$ parameters for $\pi p$ and $p p$ is too small to be observed. Indeed, both $\sigma_{0}$ and $\sigma_{1}$ for $\pi p$ collisions are about a factor of $2 / 3$ smaller than for $p p$, and this difference cancels in (16).

For heavier flavors a steeper energy dependence should be expected. For instance, in the case of $J / \Psi$-proton scattering, $\sigma_{0}$ is so small that $\epsilon \approx \Delta$. Indeed, data for $J / \Psi$ photoproduction from HERA [22] show that $\epsilon \approx$ 0.2 . One should be careful, however, in interpreting the data within the vector dominance model [23], and one should also remember that Eq. (15) was derived assuming that $r_{0}$ is much smaller than the hadronic size; otherwise interferences should be included.
The $x$ dependence of the proton structure function $F_{2}^{p}\left(x, Q^{2}\right)$ at small $Q^{2}$ also allows one to observe the expected nonuniversality of $\epsilon$. We expect $\sigma_{0}$ to fall as

$$
\sigma_{0}\left(Q^{2}\right) \approx \frac{\sigma_{0}}{1+Q^{2} / \Lambda_{\mathrm{QCD}}^{2}},
$$

where $\sigma_{0} \approx \sigma_{\mathrm{tot}}^{\rho p} \approx \sigma_{\mathrm{tot}}^{\pi p}$. Correspondingly, $\epsilon$ given by (16) first rises with $Q^{2}$, then levels off. Of course, at $Q^{2} \gg$ $\Lambda_{\mathrm{QCD}}^{2}$, when the transverse size of the $\bar{q} q$ dipole in the virtual photon $r_{T}^{2} \sim 1 /\left(m_{q}^{2}+Q^{2} / 4\right)$ becomes as small as $r_{0}^{2}$, our approach and Eq. (16) break down.

Notice that besides gluon bremsstrahlung other mechanisms of pure nonperturbative origin may also contribute to the energy dependence of hadronic cross sections. However, the models proposed in $[24,25]$ were not successful phenomenologically; the energy dependence was found to be too slow even before applying unitarity corrections. Moreover, the smaller intercept $\epsilon=0.05$ was found in [26].

\section{WHY $\alpha_{\mathbb{P}}^{\prime}$ IS SMALL, BUT $\alpha_{\mathbb{R}}^{\prime}$ IS LARGE}

One of the early achievements of the Regge-pole model was the prediction that the slope of the elastic differential

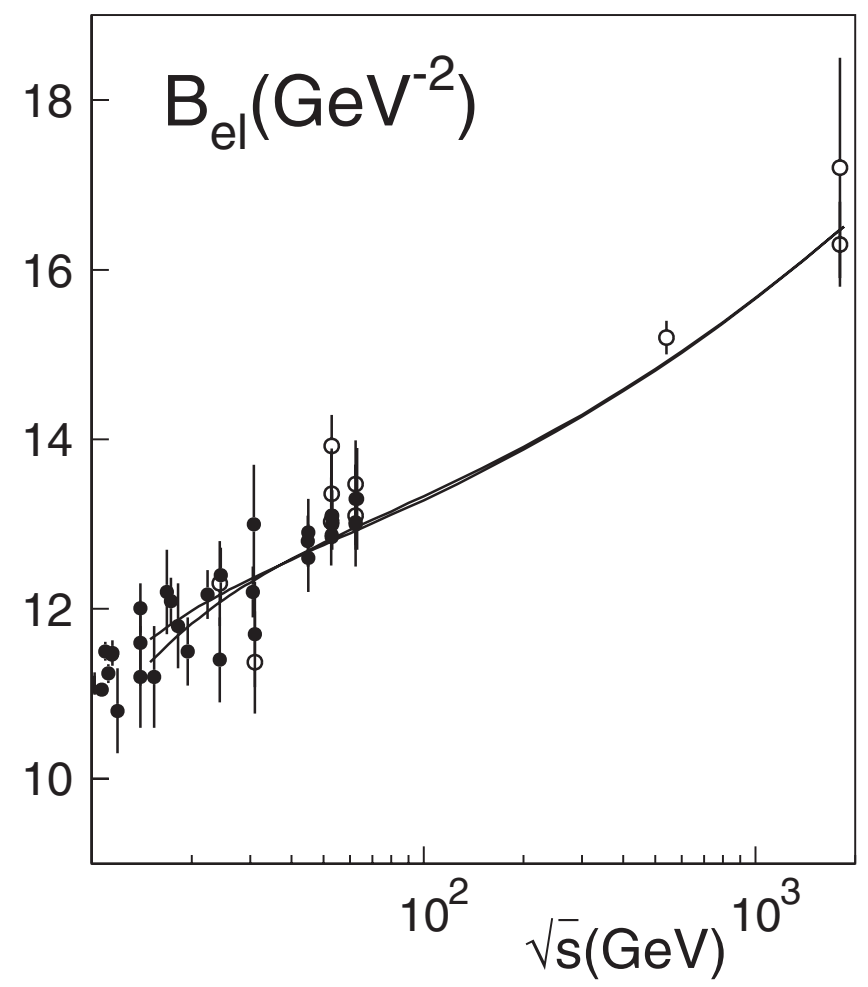

FIG. 4. Slope of elastic $p p$ and $\bar{p} p$ differential cross sections as functions of energy. The dashed and solid curves correspond to Eq. (11) with $\alpha_{\mathbb{P}}^{\prime}=0.25 \mathrm{GeV}^{-2}$ and to the slope predicted in [17] including the effects of unitarity saturation, respectively. For references to the depicted experimental data see [17]. 
cross section should rise linearly with $\ln (s)$, as in Eq. (11). This has been confirmed by data (see Fig. 4).

In terms of the parton model such a shrinkage of the diffractive cone looks like a result of Gribov's diffusion, i.e. Brownian motion of cascading partons in the impact parameter plane. If gluons are localized within a small area, i.e. they have a rather large mean transverse momentum, this may substantially slow down the diffusion. Indeed, it was found in [17] that the result of such diffusion is rather weak,

$$
\alpha_{\mathbb{P}}^{\prime}=\frac{\alpha_{s}}{3 \pi} r_{0}^{2} \approx 0.1 \mathrm{GeV}^{-2} .
$$

This value is quite below the well-known one, $\alpha_{\mathbb{P}}^{\prime}=$ $0.25 \mathrm{GeV}^{-2}$, suggested by data shown in Fig. 4. However, one should be careful with Regge-pole approximations in the case of soft hadronic interactions.

\section{A. Effects of saturation in $p p$ elastic scattering}

Since the Pomeron pole amplitude rises with energy as $f_{\text {el }}(b, s) \propto s^{\epsilon}$, it will eventually hit the unitarity limit and break the Froissart bound. However, when the interaction becomes very strong it starts screening not only specific channels, like in (9), but also itself. It has been well known since the 1970s how Regge cuts, or absorptive corrections, restore unitarity [27]; e.g. in the eikonal approximation,

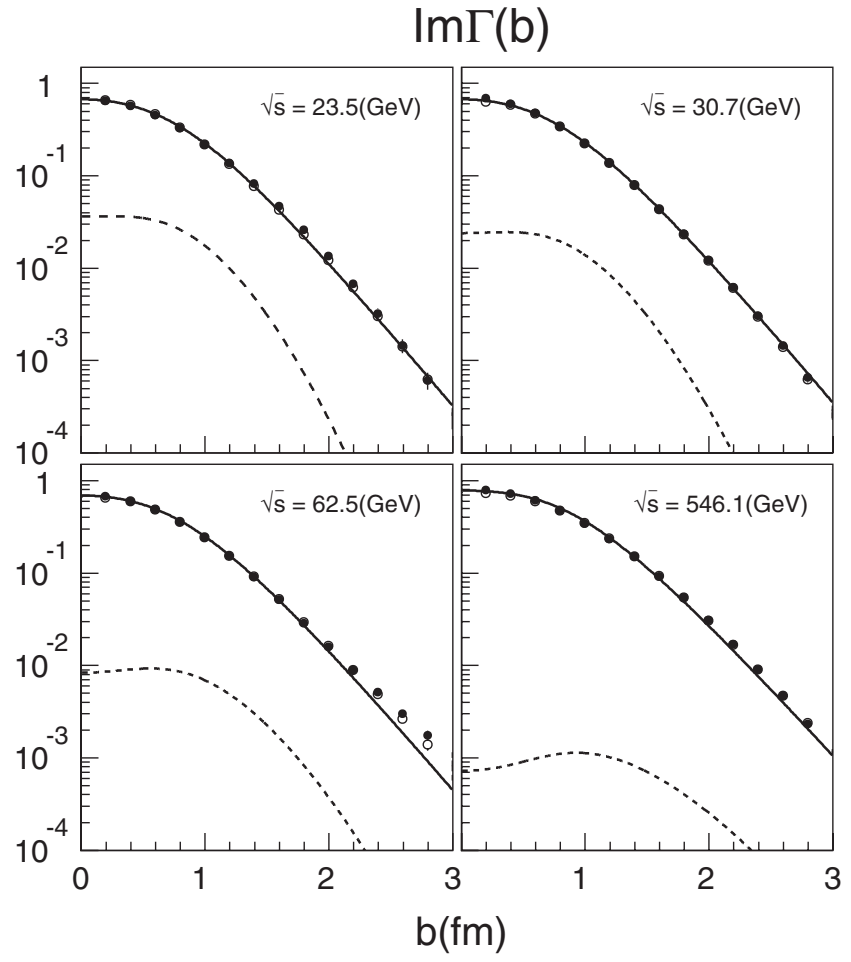

FIG. 5. Data points are Fourier transformed data for the differential elastic cross section [17]. Solid curves show the unitarized elastic amplitude, including Gribov inelastic corrections [55]. Dashed curves correspond to the Reggeon contribution, corrected for absorption. one should replace

$$
\operatorname{Im} f_{\mathrm{el}}(b, s) \Rightarrow 1-\exp \left[-\operatorname{Im} f_{\mathrm{el}}(b, s)\right] .
$$

Even if the Pomeron slope was zero, $\alpha_{\mathbb{P}}^{\prime}=0$, when the amplitude reaches the unitarity bound at small impact parameters, this area rises as a function of energy; i.e. the black disk radius squared grows proportionally to $\ln (s)$ (amazingly, like a Regge pole). The effective slope in the Froissart regime reads

$$
\alpha_{\text {eff }}^{\prime}=\frac{1}{4} \epsilon B_{0}=0.22 \mathrm{GeV}^{-2} \text {, }
$$

which is close to the one observed in data, Fig. 4. We use here $B_{0}=8.9 \mathrm{GeV}^{-2}$, from Eq. (11).

Proton-proton scattering at the highest accessed energies is close to this regime, which is something that can be seen from Fig. 5, where experimental points are Fouriertransformed data for the elastic differential cross section (see details in [17]). The solid curves show the unitarized elastic amplitude with a small Pomeron slope, Eq. (18). The energy dependent slope corresponding to this amplitude is depicted by the solid curve in Fig. 4. Apparently, it provides a correct value of $\alpha_{\text {eff }}^{\prime}$, since agreement with data is good.

\section{B. Far from saturation: electroproduction of vector mesons}

How does one disentangle the effects of unitarity saturation and the genuine Pomeron slope, Eq. (18)? For this, one should switch to a process which is not affected by the closeness of the unitarity bound. Elastic scattering of a dipole which is considerably smaller than the proton would be a proper case. Indeed, neglecting the real part of the amplitude and assuming exponential $t$ dependence, the partial amplitude for central collisions $(b=0)$ reads

$$
\operatorname{Im} f_{\mathrm{el}}(0, s)=\frac{\sigma_{\mathrm{tot}}}{4 \pi B_{e l}} .
$$

For $p p$ collisions this amplitude is slightly below the unitarity limit, since $\operatorname{Im} f_{\mathrm{el}}(b=0)<1$. For $J / \Psi$-proton elastic scattering, however, the cross section is about an order of magnitude less than $\sigma_{\text {tot }}^{p p}$ [28], but the slope is only twice as small as $B_{\mathrm{el}}^{p p}$. Thus, we expect $\operatorname{Im} f_{\mathrm{el}}^{J / \Psi p}(0, s) \approx$ 0.2 at an energy of the order of $\sqrt{s} \sim 100 \mathrm{GeV}$. This value is safely far below the unitarity limit; therefore one should expect no influence of saturation on the value of $\alpha_{\mathbb{P}}^{\prime}$, which should be as small as given by Eq. (18).

Therefore, to get rid of unitarity corrections one can consider the interaction of a small dipole with a proton, which can be made small if it involves heavy flavors, for instance, in photoproduction of heavy quarkonium. In this case the elastic amplitude is also too small to be affected by unitarity (absorptive) corrections. Then the energy dependence of the slope may be solely due to the rise of the gluon 


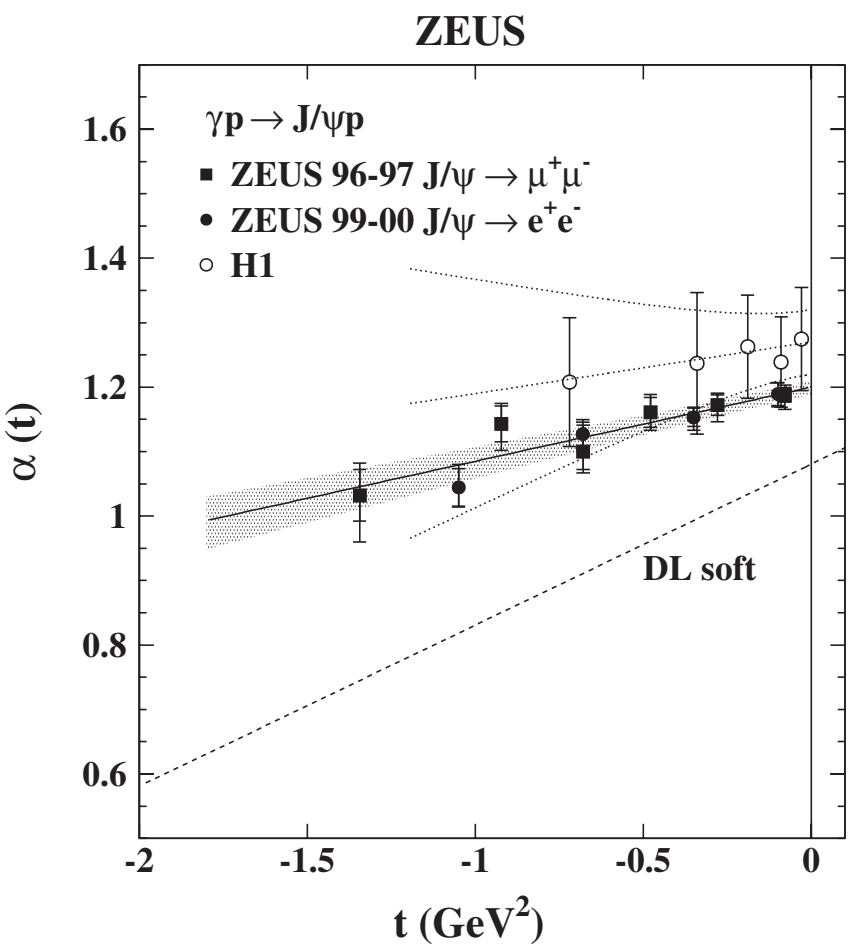

FIG. 6. The effective Pomeron trajectory measured in elastic photoproduction $\gamma p \rightarrow J / \Psi p$ [22]. The slope of the trajectory is $\alpha_{\mathbb{P}}^{\prime}=0.115 \pm 0.018 \mathrm{GeV}^{-2}$

clouds. The data for the effective Pomeron trajectory, depicted in Fig. 6, indeed demonstrate a small slope.

Another way of producing a small dipole is through virtual photoproduction of light vector mesons at high $Q^{2}$. Indeed, data depicted in Fig. 7 demonstrate a small slope parameter in photoproduction of $\rho$ mesons at high $Q^{2}$, which tends to rise towards smaller virtualities.

Here is the list of recent data for the Pomeron slope parameter, measured in different diffractive channels at HERA.

(i) $\gamma p \rightarrow J / \Psi p,[22,29]$ :

$$
\begin{aligned}
& \alpha_{\mathbb{P}}^{\prime}=0.115 \pm 0.018(\text { ZEUS }) ; \\
\alpha_{\mathbb{P}}^{\prime}= & 0.164 \pm 0.028 \pm 0.030(\mathrm{H} 1) .
\end{aligned}
$$

(ii) $\gamma^{*} p \rightarrow \rho^{0} p, Q^{2}=10 \mathrm{GeV}^{2}$ [30] (ZEUS):

$$
\begin{aligned}
& \alpha_{\mathbb{P}}^{\prime}=0.185 \pm 0.042_{-0.057}^{+0.022}\left(Q^{2}=3 \mathrm{GeV}^{2}\right) \\
& \alpha_{\mathbb{P}}^{\prime}=0.114 \pm 0.043_{-0.024}^{+0.026}\left(Q^{2}=10 \mathrm{GeV}^{2}\right)
\end{aligned}
$$

(iii) $\gamma^{*} p \rightarrow \phi p,[31]$ (ZEUS):

$$
\alpha_{\mathbb{P}}^{\prime}=0.08 \pm 0.09 \pm 0.08\left(Q^{2}=5 \mathrm{GeV}^{2}\right) \text {. }
$$

The first and second errors are statistic and systematic, respectively.
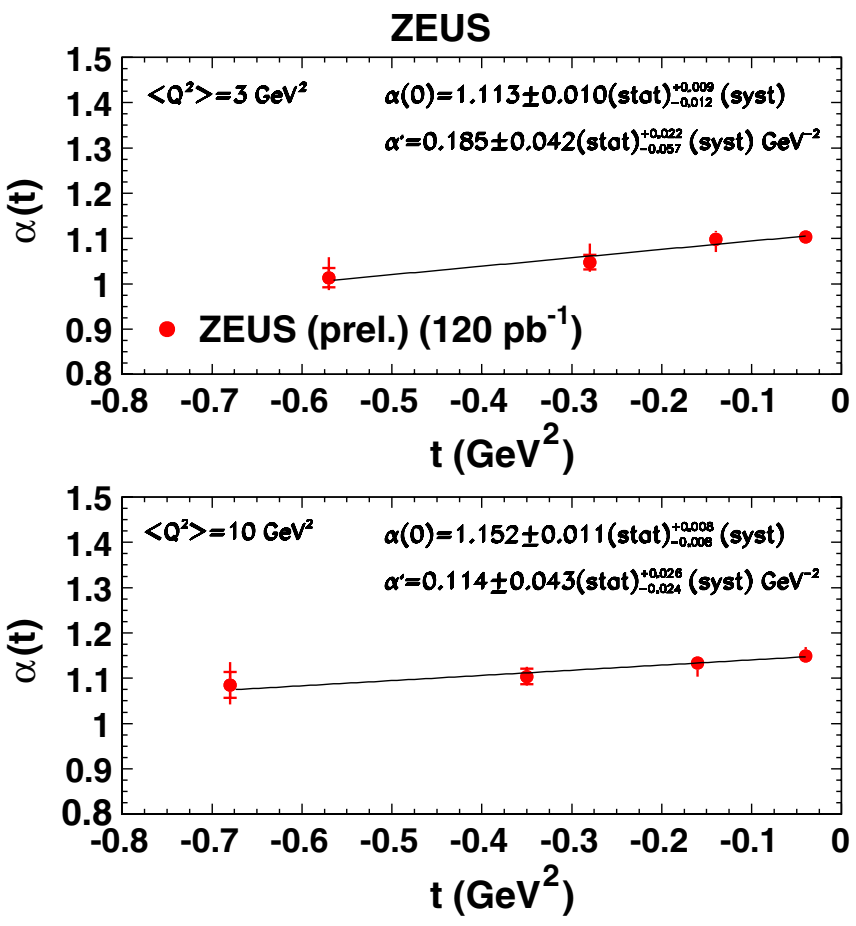

FIG. 7 (color online). The effective Pomeron trajectory measured in elastic photoproduction $\gamma p \rightarrow \rho p$ [30].

\section{Why $\boldsymbol{\alpha}_{\mathbb{R}}^{\prime} \gg \boldsymbol{\alpha}_{\mathbb{P}}^{\prime}$}

The parameter $\alpha_{\mathbb{P}}^{\prime}$ controlling the energy dependence of the interaction radius is related to the branching process of gluon radiation by gluons. Since each impact parameter step of such a branching is small, $\Delta b \sim r_{0}$, the resulting expansion is slow, and $\alpha_{\mathbb{P}}^{\prime}$, according to (18), is small also. The slope of a Regge trajectory $\alpha_{\mathbb{R}}^{\prime}$ is related to gluon emission by a valence quark which also performs Brownian motion in impact parameters. It is known from data that $\alpha_{\mathbb{R}}^{\prime} \approx 1 \mathrm{GeV}^{-2} \gg \alpha_{\mathbb{P}}^{\prime}$. At first sight, the Brownian motion of a valence quark should be as slow as for gluons, since the quark-gluon separation is small for both of them. However, there are some differences.

In the case of Pomeron exchange, in every splitting $q \rightarrow$ $q g$ or $g \rightarrow g g$ the gluon should be radiated with a small fractional momentum $\alpha \ll 1$; otherwise it would be a next-to-leading $\log (1 / x)$ correction. Such a small $\alpha$ gluon makes a short step in impact parameters, $\Delta b_{g} \sim r_{0}$, according to previous considerations.

In the case of Reggeon exchange, in the splitting $q \rightarrow q g$ the gluon is radiated with fractional momentum $\alpha \sim 1$, while the recoil valence quark gets a small fraction, $1-$ $\alpha \ll 1$. In this case the quark-gluon separation is shared differently: the gluon shift, $\Delta b_{g}=(1-\alpha) r_{T}$, is vanishing, and the quark makes a maximal step in impact parameters, $\Delta b_{q} \approx r_{T}$. The light-cone $r_{T}$-distribution amplitude is also different from Eqs. (2) and (3) which assumed $\alpha \ll 1$. For finite $\alpha \rightarrow 1$, instead of (3) one gets 


$$
\left.\Psi_{q g}^{T}(\alpha, \vec{r})\right|_{\alpha \rightarrow 1}=\frac{1}{\pi} \sqrt{\frac{\alpha_{s}}{3}} \chi_{f} \hat{\Gamma} \chi_{i} K_{0}\left(\tau r_{T}\right),
$$

where $\chi_{i, f}$ are the spinors corresponding to the initial and final quarks, and the operator $\hat{\Gamma}$ has the form $[2,14]$

$$
\hat{\Gamma}=i m_{q} \vec{e}^{*} \cdot(\vec{n} \times \vec{\sigma})+\overrightarrow{e^{*}} \cdot(\vec{\sigma} \times \vec{\nabla})-i \overrightarrow{e^{*}} \cdot \vec{\nabla} .
$$

The mean transverse quark-gluon separation squared is $\left\langle r_{T}^{2}\right\rangle=1 / \tau^{2}$, where

$$
\tau^{2}=\alpha^{2} m_{q}^{2}+\left.(1-\alpha) m_{g}^{2}\right|_{\alpha \rightarrow 1}=m_{q}^{2} .
$$

Thus, differently from Eq. (3), $\left\langle r_{T}^{2}\right\rangle$ depends on the quark, rather than the gluon mass; i.e. it is much bigger, $\left\langle r_{T}^{2}\right\rangle \gg$ $r_{0}^{2}$. Correspondingly, the slope of the Regge trajectory is larger than the Pomeron one, Eq. (18),

$$
\frac{\alpha_{\mathbb{P}}^{\prime}}{\alpha_{\mathbb{R}}^{\prime}}=r_{0}^{2} \Lambda_{\mathrm{QCD}}^{2}
$$

Here we assumed that the effective quark mass is of the order of the inverse confinement radius, $m_{q} \approx \Lambda_{\mathrm{QCD}}$. Thus, we get the Reggeon slope $\alpha_{\mathbb{R}}^{\prime} \approx 1 \mathrm{GeV}^{-2}$ in very good accord with data. We conclude that the relation between the Reggeon and Pomeron trajectories slopes is a direct consequence of the presence of two scales in the hadronic structure.

\section{FROM HARD TO SOFT REGIMES: TWO TRANSITION SCALES}

The logarithmic $Q^{2}$ derivative of the structure function $F_{2}\left(x, Q^{2}\right)$ at high $Q^{2}$ serves as a measure of the gluon density in the proton. Therefore, one should expect a steep growth of this derivative towards small $x$. Moreover, this growth should slow down considerably at some very small $x$, due to the saturation of the gluon density, which reaches equilibrium and cannot rise any more. Indeed, such an expectation was confirmed by ZEUS data [32], plotted in Fig. 8 (frequently called the Caldwell plot). The derivative $\partial F_{2}\left(x, Q^{2}\right) / \partial \ln Q^{2}$ indeed rises in accordance with Dokshitzer-Gribov-Lipatov-Altareli-Parisi (DGLAP) evolution down to $x \sim 10^{-3}$, then levels off and eventually steeply drops at $x<10^{-4}$. It is not a surprise that this observation was interpreted right away [33] as a manifestation of gluon saturation.

One should be cautious, however, treating this as small- $x$ data. Such very small values of $x$ are reached here not by means of increasing energy and keeping $Q^{2}$ constant, but by decreasing $Q^{2}$. Certainly, one can access any tiny value of $x$ by decreasing $Q^{2}$, but $x$ is not a proper variable at small $Q^{2}$ (otherwise one could explore a wonderful range of small- $x$ physics at Jefferson Lab).

\section{A. Fluctuating photon at the soft transition scale}

In the perturbative regime a virtual photon fluctuates to a $\bar{q} q$ pair with a lifetime
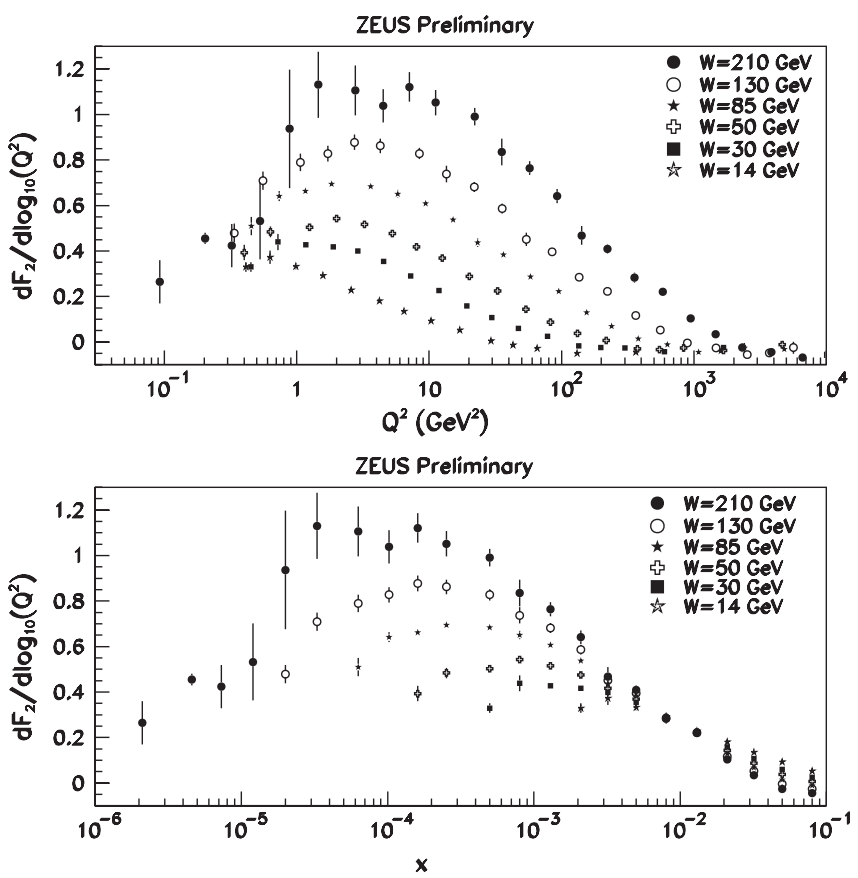

FIG. 8. Logarithmic scale derivative of the structure function as a function of $Q^{2}$ and $x$.

$$
t_{c} \sim \frac{2 \nu}{M_{\bar{q} q}^{2}+Q^{2}},
$$

where $\nu$ is the photon energy, $M_{\bar{q} q}^{2}=\left(k_{T}^{2}+m_{q}^{2}\right) / \alpha(1-\alpha)$ is the $\bar{q} q$ invariant mass squared, and $k_{T}$ and $\alpha$ are the quark transverse and fractional longitudinal momenta, respectively.

Perturbatively one treats quarks as free particles. How far can $q$ and $\bar{q}$ fly apart during the lifetime, Eq. (31)? The transverse speed of a quark of energy $\alpha \nu$ is $v_{T}^{q}=k_{T} / \alpha \nu$ (neglecting the quark mass). Therefore, after $q$ and $\bar{q}$ are produced, their separation rises during the lifetime and reaches the value

$$
r_{T}=t_{c}\left(v_{T}^{q}+v_{T}^{\bar{q}}\right)=\frac{2 k_{T}}{k_{T}^{2}+Q^{2} \alpha(1-\alpha)} .
$$

The mean value of the transverse momentum is given by the energy denominator of the fluctuation, having the same form as in (31), $\left\langle k_{T}^{2}\right\rangle=Q^{2} \alpha(1-\alpha)$. Then, from Eq. (32) we arrive at the mean $\bar{q} q$ separation squared,

$$
\left\langle r_{T}^{2}\right\rangle=\frac{1}{Q^{2} \alpha(1-\alpha)} .
$$

Although this heuristic derivation looks classical, it involves quantum effects via the finite fluctuation lifetime, Eq. (31), which arises from interferences. A full quantummechanical description of the time evolution of the fluctuation is presented in [2]. The resulting distribution function is well known [34], and it is proportional to the modified Bessel function $K_{0,1}(\epsilon r)$, where $\epsilon^{2}=Q^{2} \alpha(1-$ $\alpha)+m_{q}^{2}$. This is simply a Fourier transform of the energy 
denominator. The mean transverse separation of the $\bar{q} q$ is $\left\langle r_{T}^{2}\right\rangle=1 / \epsilon^{2}$, which is the same as in (33) if the quark mass is neglected.

The variation of the dipole size with $Q^{2}$, Eq. (33), generates the $Q^{2}$ dependence of the structure function, which describes quite well the large $Q^{2}$ data presented in Fig. 8.

At high $Q^{2}$ one can rely on asymptotic freedom and treat quarks as free particles. This is a key assumption of perturbative QCD (pQCD) . However, in the limit of real photoabsorption, $Q^{2} \rightarrow 0$, this is not appropriate any more. In this case the quarks are moving within a lightcone potential controlling the mean transverse separation of $\bar{q} q$, which is independent of $Q^{2}$ (this explains the success of vector dominance). Thus, the logarithmic $Q^{2}$ derivative of $F_{2}$ must vanish at $Q^{2} \rightarrow 0$, and this indeed is confirmed by the data depicted in Fig. 8 .

However, the transition scale for the onset of nonperturbative effects turns out to be too small, $Q^{2} \sim \Lambda_{\mathrm{QCD}}^{2} / \alpha(1-$ $\alpha)<0.16 \mathrm{GeV}^{2}$. This is much smaller than the values $Q^{2} \sim 2-4 \mathrm{GeV}^{2}$ where the data depicted in Fig. 8 deviate from the $\mathrm{pQCD}$ predictions.

\section{B. Semihard transition scale}

The smallness of the mean radius of gluon propagation, $r_{0}$, should not affect the proton structure function at small $x \ll 1$ and large $Q^{2} \gg 4 / r_{0}^{2}$. In fact, the size of the gluon cloud of a $\bar{q} q$ fluctuation of the virtual photon is small anyway, smaller than $r_{0}$, since it is cut off by color screening in the same way as in the case of color transparency. However, when the mean dipole separation $2 / Q$ becomes larger than $r_{0}$, the interference between gluon radiation by $q$ and $\bar{q}$ weakens, and they acquire independent (and $Q^{2}$ independent) gluon distributions. Thus, the presence of the semihard scale $1 / r_{0}$ leads to a turnover in the $Q^{2}$ dependence of the structure function at $Q^{2} \sim 4 / r_{0}^{2} \approx 1.8 \mathrm{GeV}^{2}$.

At smaller $Q^{2}$, or larger dipole separation, the dipole cross section is believed to level off. Using the popular parametrization from [35], we get

$$
\begin{aligned}
\frac{d \sigma\left(Q^{2}\right)}{d \ln \left(Q^{2}\right)} & \sim \frac{d \sigma(r)}{d \ln \left(r^{2}\right)}=\frac{1}{4} \sigma_{0} r^{2} Q_{s}^{2}(x) e^{-r^{2} Q_{s}^{2}(x) / 4} \\
& \sim \sigma_{0} \frac{Q_{s}^{2}(x)}{Q^{2}} e^{-Q_{s}^{2}(x) / Q^{2}} .
\end{aligned}
$$

Thus, the logarithmic derivative stops rising at $Q^{2} \sim Q_{s}^{2} \approx$ $1 \mathrm{GeV}^{2}$ and then starts falling down. This is in fair agreement with the above estimate.

One can rephrase this in the momentum representation: when the $\bar{q} q$ relative transverse momentum, $k_{T}^{q} \sim Q / 2$, is much smaller than the generic transverse momentum of gluons, $k_{T}^{g} \sim 1 / r_{0}$, the variation of $Q$, i.e. of the dipole size, does not affect the gluon radiation any more. The dipole cross section levels off at large separation, leading to a reduction of the logarithmic derivative, Eq. (34).
The observed behavior of the logarithmic $Q^{2}$ derivative of $F_{2}\left(x, Q^{2}\right)$ has been interpreted in terms of low- $Q^{2}$ behavior, rather than low- $x$ behavior, in Ref. [36]. However, a semihard scale $Q_{0}^{2}=2 \mathrm{GeV}^{2}$ was, in this case, introduced with no motivation.

\section{Variation of the energy dependence with the scale}

One of the main results of HERA is the observation of a steep rise with $Q^{2}$ of the energy slope $\lambda_{\text {eff }}\left(Q^{2}\right)=$ $d \ln \left(F_{2}\right) / d \ln (s)$, which is well explained by DGLAP evolution. On the other hand, one expects $\lambda$ to approach the known hadronic value $\lambda=0.1$ in the soft limit $Q^{2} \rightarrow 0$. At which value of $Q^{2}$ does this transition happen? The change of regime should occur for the same reason as was discussed above for the logarithmic $Q^{2}$ derivative, namely, when the mean $\bar{q} q$ separation exceeds the mean size of the gluonic clouds, $r_{0} \approx 0.3 \mathrm{fm}$. Therefore it is expected to happen at the same semihard scale. ZEUS data for $\lambda_{\text {eff }}\left(Q^{2}\right)$, presented in Fig. 9, confirm this.

Notice that this two-scale behavior is different from the model [37] of soft and hard Pomerons. Both terms in Eq. (15) effectively correspond to the soft Pomeron of [37]. At $Q^{2} \gg 1 / r_{0}^{2}$ the semihard scale $r_{0}$ has no influence and the energy dependence can be described by the DGLAP Pomeron. This regime is beyond the scope of present consideration.

\section{ZEUS 1995}

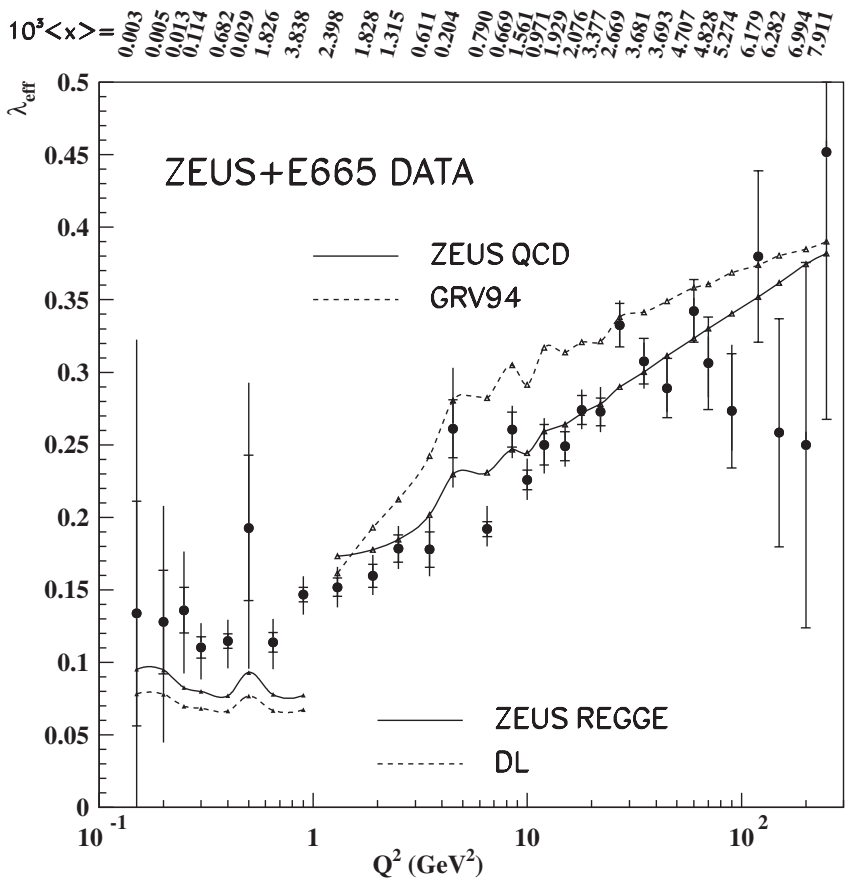

FIG. 9. $\quad \lambda_{\text {eff }}=d \ln F_{2} / d \ln (1 / x)$ as a function of $Q^{2}$, calculated in [58] by fitting $F_{2}=A x^{-\lambda_{\text {eff }}}$ to ZEUS and E665 data with $x<$ 0.01 . The DL and GRV94 calculations are from Ref. [59] and the GRV94 NLO fit [60], respectively. 


\section{FERMI MOTION OF QUARKS AND GLUONS}

At a soft scale the interaction does not resolve the gluonic structure of a hadron, but only the presence of the valence quarks. The mean transverse Fermi momentum of these quarks is small,

$$
k_{T}^{v} \sim \frac{1}{r_{h}} \sim \Lambda_{\mathrm{QCD}}
$$

At a higher scale relevant to hard reactions, one can resolve the structure of the valence quarks, i.e. the presence of gluons and sea quarks. Since these are located at small separations, $\sim r_{0}$, from the valence quark, both have more intensive intrinsic Fermi motion,

$$
\left\langle k_{0}^{2}\right\rangle \sim \frac{1}{r_{0}^{2}} .
$$

Such a large primordial momentum should affect the onset of the hard regime in the particular process.

\section{A. Cronin effect}

A projectile parton propagating through a nucleus experiences multiple interactions, increasing its transverse momentum. Then the parton participating in a hard collision inside the nucleus has an increased transverse momentum compared to Eq. (36), which corresponds to the interaction with a free proton,

$$
\left\langle k_{A}^{2}\right\rangle=\left\langle k_{0}^{2}\right\rangle+\Delta k^{2},
$$

where $\Delta k^{2}$ is the nuclear broadening.

This observation helps one to understand the Cronin effect [38] of nuclear enhancement of hadron production with high $p_{T}$. Apparently, the strength of the effect depends on the relative values of the two terms in (37). In the limit of a weak primordial motion the effect should be strongest, while in the case of $\left\langle k_{0}^{2}\right\rangle \gg \Delta k^{2}$ the effect will disappear.

A rather strong Cronin effect was observed in fixed target experiments, where production of high- $p_{T}$ hadrons is dominated by the scattering of valence quarks [8] which have a small primordial $k_{T} \sim 1 \mathrm{fm}^{-1}$. One can access the gluons only at sufficiently high energies. Relying on the above consideration, a very weak Cronin enhancement was predicted in [8] at $\sqrt{s}=200 \mathrm{GeV}$, as is depicted in Fig. 10. A several times stronger effect was predicted in [39], ${ }^{1}$ and a suppression, rather than an enhancement, was the expectation of the color glass condensate model [42]. The latest data from the PHENIX experiment at RHIC, depicted in Fig. 10, confirm the prediction of [8].

It is worth mentioning that interferences, which are usually assumed to be negligible for hard reactions on

\footnotetext{
${ }^{1}$ The extremely strong gluon shadowing implemented into the HIJING model is ruled out by the recent LO [40] and NLO analyses [41] of DIS data.
}

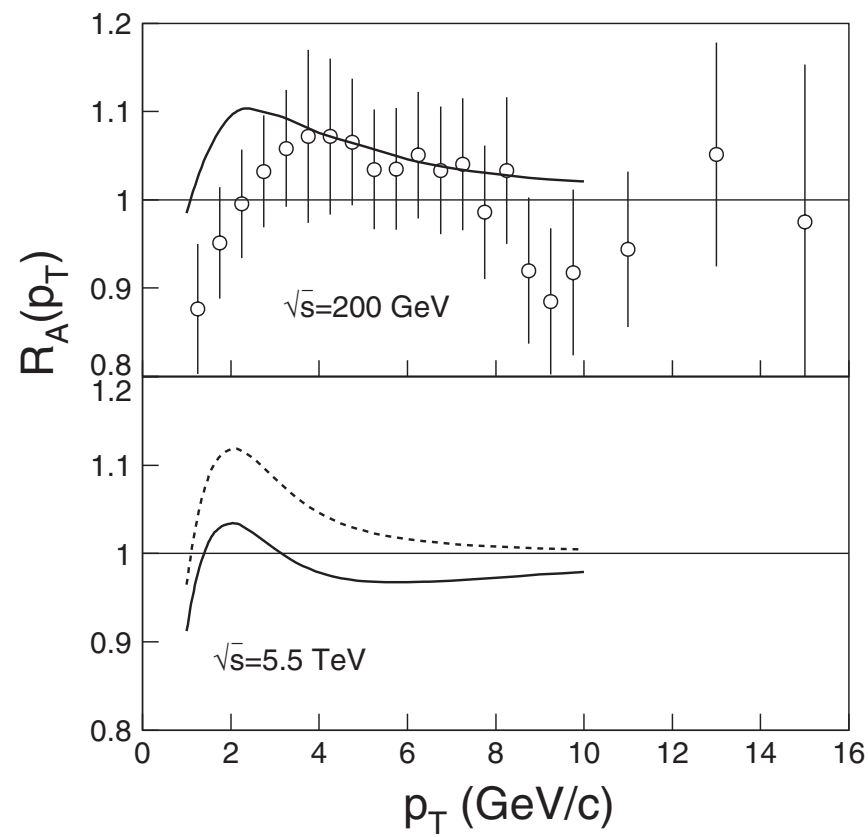

FIG. 10. Nucleus-to-proton cross section ratio for pion production, versus $p_{T}$. Dashed and solid curves correspond to calculations without or with gluon shadowing [8].

nuclei, become important if the collision energy is sufficiently high. In this case the process is called coherent. The mechanisms of the Cronin enhancement are quite different in these two limiting cases. In the case of incoherent hard interaction the incoming projectile partons (mainly valence quarks) experience initial state multiple soft rescattering leading to a high- $p_{T}$ enhancement, and the projectile gluons located in small spots are not resolved. If, however, the energy is sufficiently high, the lifetime of the hard fluctuation exceeds the nucleus size and the process becomes coherent. In this case gluons are well resolved, since they

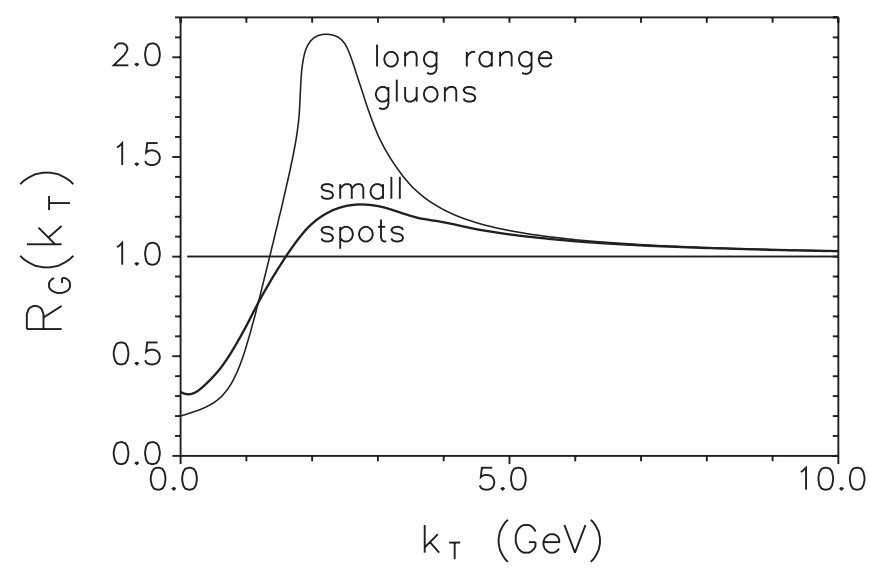

FIG. 11. Nucleus-to-proton cross section ratio for gluon radiation as a function of $p_{T}$. The upper and lower curves correspond to mean gluon propagation distances $1 \mathrm{fm}$ (long range) and $0.3 \mathrm{fm}$ (small spots), respectively [14]. 
dominate the production of high- $p_{T}$ partons. Such a coherent regime is relevant for hadron production at moderately large $p_{T}$ at RHIC, and it dominates a large range of $p_{T}$ at CERN LHC energies. Besides, in the latter case the Cronin effect is substantially reduced by shadowing (see the next section), as is demonstrated in Fig. 10.

In the coherent regime the interaction resolves the gluons and their primordial transverse momentum is of great importance: the larger it is, the smaller the Cronin enhancement. This is demonstrated in Fig. 11 for coherent gluon radiation by a quark propagating through a nucleus. The depicted ratio of the radiation cross sections on nucleus-toproton targets is calculated with different assumptions about the mean propagation length of gluons. The upper curve corresponds to a long range propagation $\sim 1 \mathrm{fm}$, while the bottom one assumes that gluons are confined within small spots $\sim 0.3 \mathrm{fm}$. In the latter case the gluon primordial momentum is larger; correspondingly the Cronin enhancement is much weaker in good accord with RHIC data shown in Fig. 10.

\section{B. Other processes}

The mean primordial transverse momentum of projectile partons can be generated perturbatively. Namely, higher order processes include gluon bremsstrahlung, which leads

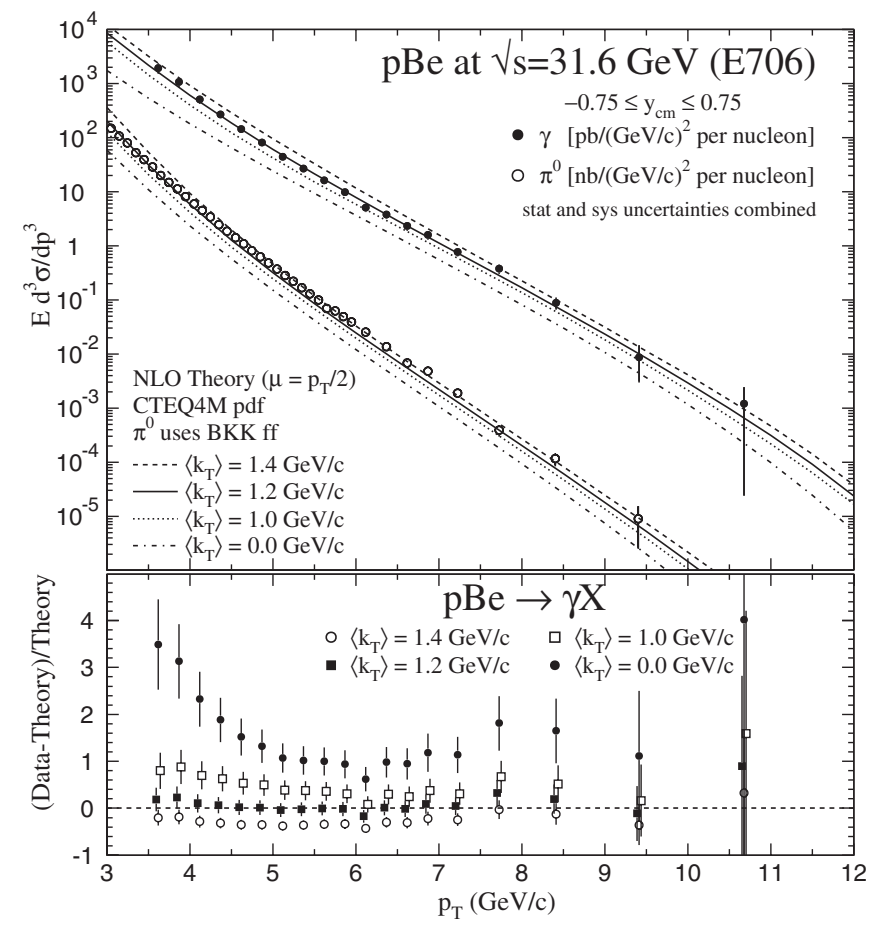

FIG. 12. Top plot: the photon and $\pi^{0}$ production cross sections from the E706 experiment at $\sqrt{s}=31.6 \mathrm{GeV}$, compared to $k_{T}$-corrected NLO calculations [44]. Bottom plot: the ratio (Data-Theory)/Theory for direct photon production. Theory corresponds to the NLO calculations with primordial parton momentum $\left\langle k_{T}\right\rangle$. to increasing transverse momenta of partons radiating gluons. Nevertheless, NLO calculations fail to explain data on Drell-Yan reactions [43], and direct photon [44] and heavy flavor production [45], unless an additional primordial transverse momentum $k_{T}$ is introduced. All these reactions demand a mean primordial momentum, $\left\langle k_{T}\right\rangle \approx 1 \mathrm{GeV}$.

Comparison of NLO calculations and data [44], for both direct photons and neutral pion production, is depicted in Fig. 12. The primordial momentum $\left\langle k_{T}\right\rangle \approx 1.2 \mathrm{GeV}$ seems to provide the best description of data. This value is somewhat larger than the semihard scale under discussion, but it includes contributions from terms higher than NLO. Besides, in these calculations there is no strict border between the soft part of gluon radiation responsible for $\left\langle k_{T}\right\rangle$ and the hard part ascribed to the perturbative NLO part.

Notice that in the dipole description of direct photon $[14,46]$ and Drell-Yan pair [47] production there is no need (no uncertainty either) to introduce any primordial momentum, beyond a soft one related to the hadron size. The semihard primordial momentum is included by default in the phenomenological dipole cross section.

A similar value of the primordial momentum was extracted from azimuthal angle correlations measured in high- $p_{T}$ dihadron production at RHIC [48],

$$
\sqrt{\left\langle k_{T}^{2}\right\rangle}=1.28 \pm 0.06 \mathrm{GeV}
$$

However, in this case no NLO calculation has been performed, and it is not clear which part of this primordial momentum has a nonperturbative origin.

Another observable sensitive to the primordial parton motion is the so-called seagull effect, the dependence of the mean transverse momentum of produced hadrons on Feynman $x_{F}$. The observed shape of the $x_{F}$ dependence is a result of fragmentation of forward jets [49]. Their transverse momenta include the primordial one. It was found [50] that data demand a rather large $\left\langle k_{T}\right\rangle \sim 0.5-1 \mathrm{GeV}$.

\section{NUCLEAR GLUE: SATURATED OR DILUTE?}

Gluon clouds originating from different nucleons can overlap in the longitudinal direction at small $x$, since the internucleon spacing contracts $\propto 1 / E$, while gluon clouds at small $x$ shrink as $1 / x E$. This is illustrated in Fig. 13 . Since gluons overlap they can interact and fuse, and this leads to a reduction of the number of gluons in nuclei at small $x$. This phenomenon is called gluon shadowing.

It turns out that the smallness of the gluonic spots affects the longitudinal overlap. First, it makes the clouds effectively shorter in the longitudinal direction. Indeed, the coherence length which controls the overlap of the clouds is shorter, since gluons with an enhanced transverse motion are effectively heavier. The coherence length has the form 


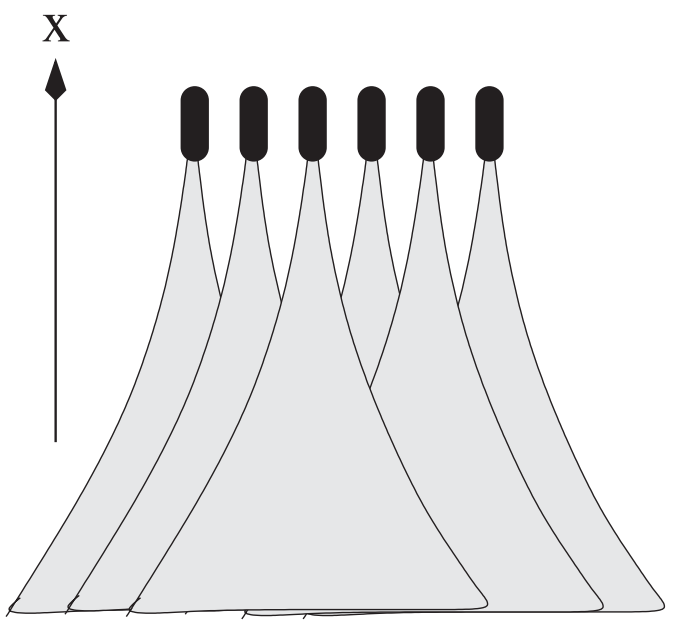

FIG. 13. Logarithmic scale derivative of the structure function as a function of $Q^{2}$ and $x$.

$$
l_{c}=\frac{P}{x m_{N}} \equiv P l_{c}^{\max },
$$

where the factor $P$ is usually assumed to be $p \approx 1 / 2$. However, even for quarks $P$ depends considerably on the polarization of the virtual photon, but for gluons it is an order of magnitude shorter [51]. One can see this in Fig. 14. Thus, gluons need much smaller $x$ than quarks to overlap in the longitudinal direction; i.e. the onset of gluon shadowing occurs at quite smaller $x$ than for quark shadowing.

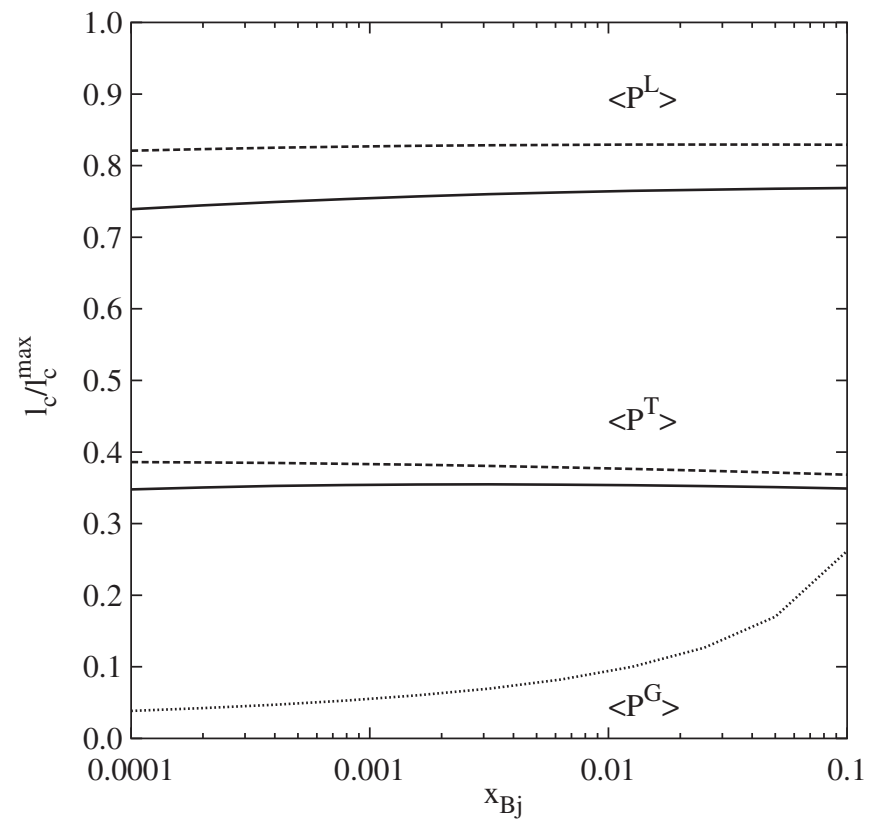

FIG. 14. Bjorken $x$ dependence of the factor $P$ in Eq. (39) for quarks and longitudinal (two upper curves) and transverse (next couple of curves) photons. Solid and dashed curves are calculated [51] for $Q^{2}=4$ and $40 \mathrm{GeV}^{2}$, respectively. The bottom curve represents $P$ for gluons.
However, it is not sufficient for gluons to overlap in the longitudinal direction; in order to interact they also have to overlap in the transverse plane. If gluon clouds are as big as the proton, the mean number of other clouds overlapping with a particular one is $\pi R_{p}^{2}\left\langle T_{A}\right\rangle$, where the mean nuclear thickness is $\left\langle T_{A}\right\rangle=1 / A \int d^{2} b T_{A}^{2}(b)$ and $T_{A}(b)=\int_{-\infty}^{\infty} d z \rho_{A}(b, z)$. For lead $\left\langle T_{A}\right\rangle=1.35 \mathrm{fm}^{-2}$, so the mean number of such overlaps is rather large, about 4.

On the other hand, if the quark-gluon separation is as small as $r_{0}$, the mean number of other dipoles overlapping transversely with this one is

$$
\langle n\rangle=\frac{3 \pi r_{0}^{2}}{4}\left\langle T_{A}\right\rangle \approx 0.3 .
$$

This shows that even at small $x$ the overlap of gluons is very small, and therefore shadowing is weak. An example is depicted in Fig. 15.

Experimental information about gluon shadowing is extremely scarce. Only one experiment, New Muon Collaboration [52], was able to detect a variation with $Q^{2}$ of nuclear effects in DIS at small $x$. Leading order DGLAP analyses [53,54] of this data were unable to extract gluon shadowing. Only NLO analyses by de Florian and Sassot [41] turned out to be sensitive to gluons. Gluon shadowing was found to be very weak, as predicted in [2], and even somewhat weaker.

Unfortunately, this evidence of gluon shadowing is based on the results of only one experiment. New measurements are desperately needed, and the eRHIC collider would offer a wonderful opportunity for this physics.

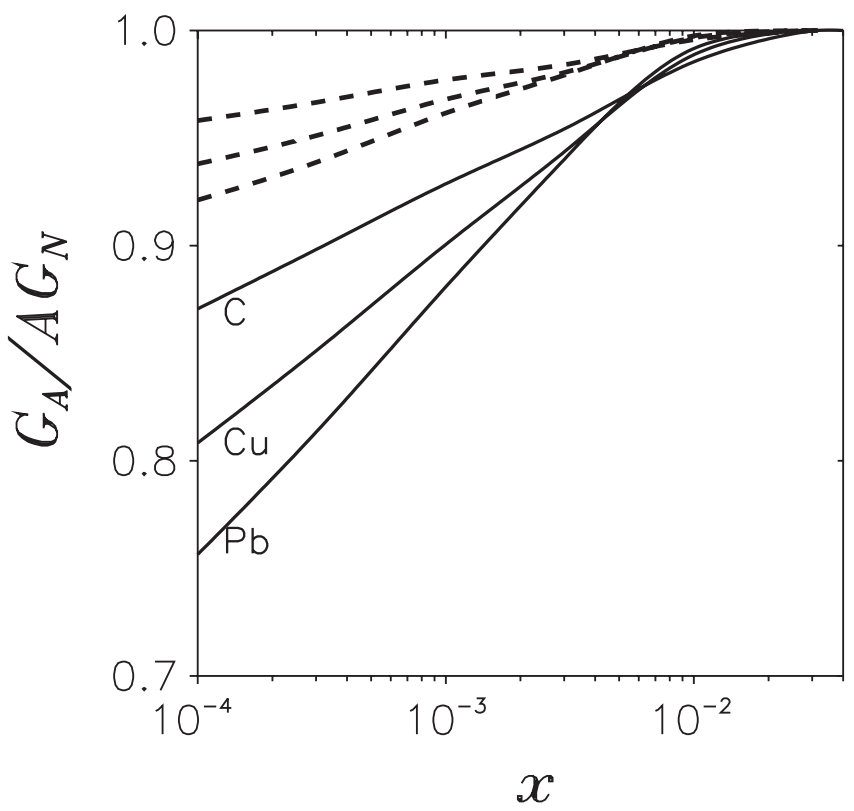

FIG. 15. Ratio of the gluon distribution functions in nuclei (carbon, copper, and lead) and nucleons versus Bjorken $x$ at $Q^{2}=4 \mathrm{GeV}^{2}$ (solid curves) and $40 \mathrm{GeV}^{2}$ (dashed curves). 


\section{SUMMARY}

The presence of the semihard scale in hadrons, corresponding to distances as small as $0.3 \mathrm{fm}$, leads to numerous observable effects. It helps to explain, from a common point of view, a series of puzzling features observed in particle collisions at high energies.

(i) Smallness of the triple-Pomeron coupling, which means that diffractive gluon radiation is suppressed.

(ii) $t$ independence of the triple-Pomeron coupling.

(iii) The small value of the Pomeron intercept, $\alpha_{\mathbb{P}}(0)-$ $1=0.1$.

(iv) The small value of the Pomeron trajectory slope, $\alpha_{\mathbb{P}}^{\prime} \approx 0.1 \mathrm{GeV}^{-2}$, observed in exclusive electroproduction of vector mesons. At the same time the unitarity saturation effects lead to a larger value, $\alpha_{\text {eff }}^{\prime}=0.25 \mathrm{GeV}^{-2}$ in $p p$ collisions. Although Brownian motion of gluons in impact parameters is very slow, valence quarks emitting gluons move much faster, and Eq. (30) reproduces well the large slope of Regge trajectories, $\alpha_{\mathbb{P}}^{\prime} \approx 1 \mathrm{GeV}^{-2}$. This relation, Eq. (30), is a direct consequence of the two-scale structure of hadrons.

(v) The two hadronic scales can be seen in the transition from hard to soft regimes in deep-inelastic scattering. The logarithmic derivative $\partial F_{2} / \partial \ln \left(Q^{2}\right)$ vanishes at very small $Q^{2}<4 \Lambda_{\mathrm{QCD}}^{2}$, but deviates from the expected DGLAP behavior at much higher values, $Q^{2} \sim 2 \mathrm{GeV}^{2}$.

(vi) The presence of the semihard scale in the primordial transverse momenta of partons leads to a very weak nuclear (Cronin) enhancement of high- $p_{T}$ hadrons observed in data at RHIC. This means that the color glass condensate is a rather weak effect in the available energy range.

(vii) Other hard reactions sensitive to the primordial parton motion (direct photon, Drell-Yan dileptons, heavy flavors, back-to-back dihadrons, seagull effect, etc.) also demand a large transverse momenta of the projectile partons, which cannot be explained by NLO calculations.

(viii) NLO analysis of nuclear structure functions demonstrates a very weak gluon shadowing which is a clear result of a strong gluon localization. This result is related to the observed weak diffractive excitation of gluonic degrees of freedom. Indeed, nuclear shadowing is directly related to diffraction via Gribov's inelastic shadowing [55].

Notice that for most experimental facts presented in this list no alternative explanation has been proposed so far. Therefore they maybe be considered as solid experimental evidences in favor of the presence of a semihard scale in hadronic structure. Still, this important issue demands further study, and new precise data are desperately needed. In this respect a planned electron-nucleus collider (eRHIC) will be a wonderful facility for these researches.

\section{ACKNOWLEDGMENTS}

We are thankful to Hans-Jürgen Pirner for interesting discussions. This work was supported in part by Fondecyt (Chile) Grant No. 1050589 and No. 1050519, and by DFG (Germany) Grant No. PI182/3-1.
[1] L. N. Lipatov, Yad. Fiz. 23, 642 (1976) [Sov. J. Nucl. Phys. 23, 338 (1976)]; V.S. Fadin, E. A. Kuraev, and L. N. Lipatov, Phys. Lett. 60B, 50 (1975); I. I. Balitsky and L. N. Lipatov, Yad. Fiz. 28, 1597 (1978) [Sov. J. Nucl. Phys. 28, 822 (1978)]; Pis'ma Zh. Eksp. Teor. Fiz. 30, 383 (1979) [JETP Lett. 30, 355 (1979)].

[2] B. Z. Kopeliovich, A. Schäfer, and A. V. Tarasov, Phys. Rev. D 62, 054022 (2000).

[3] A. DiGiacomo and H. Panagopoulos, Phys. Lett. B 285, 133 (1992).

[4] H. G. Dosch, E. Ferreira, and A. Kramer, Phys. Rev. D 50, 1992 (1994).

[5] T. Schäfer and E. V. Shuryak, Rev. Mod. Phys. 70, 323 (1998).

[6] E. Shuryak and I. Zahed, Phys. Rev. D 69, 014011 (2004).

[7] V. M. Braun, P. Górnicki, and L. Mankiewicz, Phys. Lett. B 302, 291 (1993)

[8] B.Z. Kopeliovich, J. Nemchik, A. Schäfer, and A. V. Tarasov, Phys. Rev. Lett. 88, 232303 (2002).
[9] B. Z. Kopeliovich and B. Povh, J. Phys. G 30, S999 (2004).

[10] B. Z. Kopeliovich, B. Povh, and I. Schmidt, Nucl. Phys. A782, 24 (2007).

[11] Yu. M. Kazarinov, B.Z. Kopeliovich, L. I. Lapidus, and I. K. Potashnikova, Zh. Eksp. Teor. Fiz. 43, 598 (1976) [Sov. Phys. JETP 70, 1152 (1976)].

[12] B.Z. Kopeliovich, L.I. Lapidus, and A. B. Zamolodchikov, Pis'ma Zh. Eksp. Teor. Fiz. 33, 612 (1981); [Sov. Phys. JETP 33, 595 (1981)].

[13] G. Ingelman and K. Prytz, Z. Phys. C 58, 285 (1993).

[14] B.Z. Kopeliovich, A. Schäfer, and A. V. Tarasov, Phys. Rev. C 59, 1609 (1999).

[15] U. Amaldi and K. R. Schubert, Nucl. Phys. B166, 301 (1980).

[16] B. Z. Kopeliovich, B. Povh, and E. Predazzi, Phys. Lett. B 405, 361 (1997).

[17] B.Z. Kopeliovich, I. K. Potashnikova, B. Povh, and E. Predazzi, Phys. Rev. Lett. 85, 507 (2000); Phys. Rev. D 63, 054001 (2001). 
[18] E. Gotsman, E. M. Levin, and U. Maor, Z. Phys. C 57, 677 (1993); Phys. Rev. D 49, R4321 (1994); Phys. Lett. B 353, 526 (1995); 347, 424 (1995).

[19] K. Goulianos and J. Montanha, Phys. Rev. D 59, 114017 (1999).

[20] S. Erhan and P. E. Schlein, Phys. Lett. B 427, 389 (1998).

[21] S. Eidelman et al. (Particle Data Group), Phys. Lett. B 592, 1 (2004).

[22] S. Chekanov et al. (ZEUS Collaboration), Nucl. Phys. B695, 3 (2004).

[23] J. Hüfner and B. Z. Kopeliovich, Phys. Lett. B 426, 154 (1998).

[24] D. Kharzeev and E. Levin, Nucl. Phys. B578, 351 (2000).

[25] D. E. Kharzeev, Y. V. Kovchegov, and E. Levin, Nucl. Phys. A690, 621 (2001).

[26] E. V. Shuryak, Phys. Lett. B 486, 378 (2000).

[27] M.S. Dubovikov, B.Z. Kopeliovich, L. I. Lapidus, and K. A. Ter-Martirosian, Nucl. Phys. B123, 147 (1977).

[28] J. Hüfner, Yu. P. Ivanov, B. Z. Kopeliovich, and A. V. Tarasov, Phys. Rev. D 62, 094022 (2000).

[29] A. Aktas et al. (H1 Collaboration), Eur. Phys. J. C 46, 585 (2006).

[30] S. Chekanov et al. (ZEUS Collaboration), DESY Report No. DESY-07-118.

[31] S. Chekanov et al. (ZEUS Collaboration), Nucl. Phys. B718, 3 (2005).

[32] A. Caldwell, DESY Theory Workshop, DESY, Hamburg, Germany, 1997; J. Breitweg et al. (ZEUS Collaboration), Eur. Phys. J. C 7, 609 (1999); B. Foster, Eur. Phys. J. direct C 4S1, 37 (2002).

[33] E. Gotsman, arXiv:hep-ph/9906436.

[34] J. M. Bjorken, J. B. Kogut, and D. E. Soper, Phys. Rev. D 3, 1382 (1971).

[35] K. Golec-Biernat and M. Wüsthoff, Phys. Rev. D 59, 014017 (1998).

[36] C. Merino, A. B. Kaidalov, and D. Pertermann, arXiv:hep$\mathrm{ph} / 9911331$.

[37] A. Donnachie and P. V. Landshoff, Phys. Lett. B 437, 408 (1998).

[38] D. Antreasyan et al., Phys. Rev. D 19, 764 (1979).

[39] X. N. Wang, Phys. Rev. C 61, 064910 (2000).
[40] K. J. Eskola, H. Honkanen, V. J. Kolhinen, and C. A. Salgado, Phys. Lett. B 532, 222 (2002).

[41] D. de Florian and R. Sassot, Phys. Rev. D 69, 074028 (2004).

[42] D. Kharzeev, E. Levin, and L. McLerran, Phys. Lett. B 561, 93 (2003).

[43] D. C. Hom et al., Phys. Rev. Lett. 37, 1374 (1976); D. M. Kaplan et al., Phys. Rev. Lett. 40, 435 (1978).

[44] L. Apanasevich et al., Phys. Rev. Lett. 81, 2642 (1998); Phys. Rev. D 59, 074007 (1999).

[45] M.L. Mangano, P. Nason, and G. Ridolfi, Nucl. Phys. B373, 295 (1992).

[46] B.Z. Kopeliovich, A.H. Rezaeian, H. J. Pirner, and I. Schmidt, Phys. Lett. B 653, 210 (2007).

[47] B. Z. Kopeliovich, J. Raufeisen, and A. V. Tarasov, Phys. Lett. B 503, 91 (2001).

[48] J. Rak, arXiv:nucl-ex/0306031.

[49] F. E. Close, F. Halzen, and D. M. Scott, Phys. Lett. 68B, 447 (1977).

[50] N. M. Agababyan et al. (NA22 Collaboration), Phys. Lett. B 320, 411 (1994).

[51] B.Z. Kopeliovich, J. Raufeisen, and A. V. Tarasov, Phys. Rev. C 62, 035204 (2000).

[52] P. Amaudruz et al., Nucl. Phys. B441, 3 (1995); M. Arneodo et al., Nucl. Phys. B441, 12 (1995).

[53] K. J. Eskola, V. J. Kolhinen, and P. V. Ruuskanen, Nucl. Phys. B535, 351 (1998); K. J. Eskola, V. J. Kolhinen, and C. A. Salgado, Eur. Phys. J. C 9, 61 (1999).

[54] M. Hirai, S. Kumano, and M. Miyama, Phys. Rev. D 64, 034003 (2001).

[55] V. N. Gribov, Zh. Eksp. Teor. Fiz. 56, 892 (1969) [Sov. Phys. JETP 56, 892 (1969)].

[56] A. B. Kaidalov, Phys. Rep. 50, 157 (1979).

[57] A. Brandt et al. (UA8 Collaboration), Eur. Phys. J. C 25, 361 (2002).

[58] J. Breitweg et al. (ZEUS Collaboration), Eur. Phys. J. C 7, 609 (1999).

[59] A. Donnachie and P. Landshoff, Z. Phys. C 61, 139 (1994).

[60] M. Glück, E. Reya, and A. Vogt, Z. Phys. C 67, 433 (1995). 